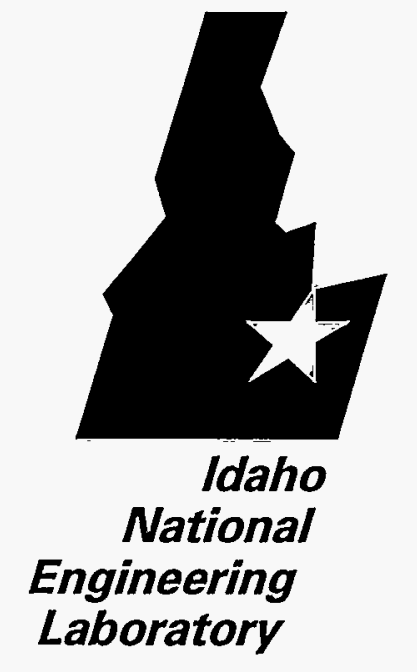

INEL-95/0120

June 1996

\section{Modeling Patterns in Data Using Linear and Related Models}

\author{
RECEIVED \\ JUL $2^{9} 1996$ \\ OSTI
}

M. E. Engelhardt

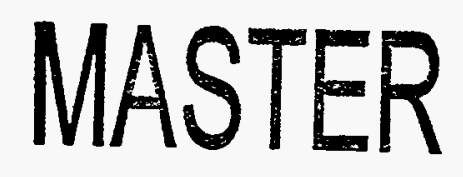

इLLockheed
Idaho Technologies Company

DISIFGUIION OF THIS DOCURENT IS UAL 


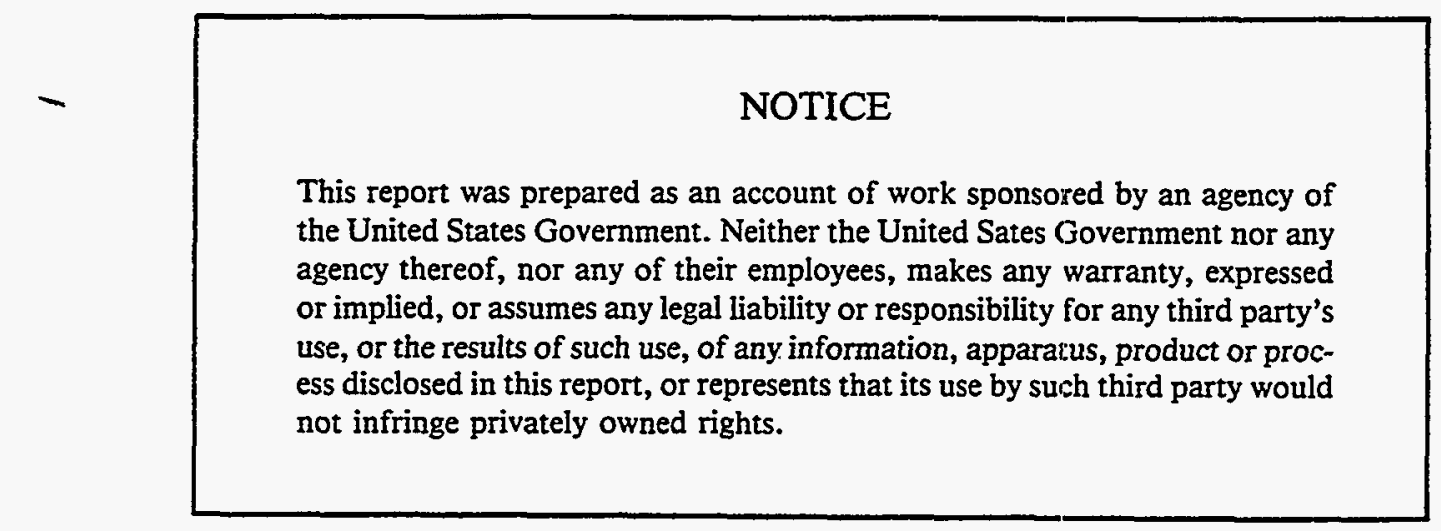




\title{
Modeling Patterns in Data Using Linear and Related Models
}

\begin{abstract}
M. E. Engelhardt
Published June 1996

Idaho National Engineering Laboratory

Idaho Falls, Idaho 83415
\end{abstract}

\author{
Prepared for the \\ U.S. Nuclear Regulatory Commission \\ Office for Analysis and Evaluation of Operational Data \\ Reliability and Risk Analysis Branch. \\ Under DOE Idaho Operations Office \\ Contract DE-AC07-94ID13223
}

DISTRIBUTION OF THIS LOCUMENT IS UNLIMRTED 


\section{DISCLAIMER}

Portions of this document may be illegible in electronic image products. Images are produced from the best available original document. 


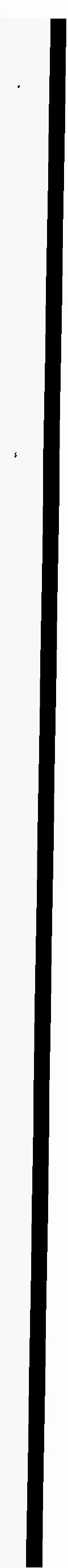




\begin{abstract}
This report considers the use of linear models, primarily linear regression and related analyses, in the analysis of operational data. In the applications considered here the covariates are related to time, either age or the year of operation. Typical examples of response variables are quantities related to reliability of systems such as failure rate or unreliability, or to public safety such as radiation exposure. An important aspect of the analysis is the study of trends, either increasing or decreasing, in time. The report shows how to fit models to data, and how to assess how well a model fits. Some examples using SAS are used to illustrate the methods.
\end{abstract}

\title{
DISCLAIMER
}

\begin{abstract}
This report was prepared as an account of work sponsored by an agency of the United States Government. Neither the United States Government nor any agency thereof, nor any of their employees, makes any warranty, express or implied, or assumes any legal liability or responsibility for the accuracy, completeness, or usefulness of any information, apparatus, product, or process disclosed, or represents that its use would not infringe privately owned rights. Reference herein to any specific commercial product, process, or service by trade name, trademark, manufacturer, or otherwise does not necessarily constitute or imply its endorsement, recommendation, or favoring by the United States Government or any agency thereof. The views and opinions of authors expressed herein do not necessarily state or reflect those of the United States Government or any agency thereof.
\end{abstract}

FIN E8247-Special Methods and Databases: Statistical Methods 


\section{SUMMARY}

This report considers the use of linear models for analyzing data related to reliability and safety issues of the type usually associated with nuclear power plants.

The report discusses some of the general results of linear regression analysis, such as the model assumptions and properties of the estimators of the parameters. The results are motivated with examples of operational data.

Results about the important case of a linear regression model with one covariate are covered in detail. This case includes analysis of time trends. The analysis is applied with two different sets of time trend data. Diagnostic procedures and tests for the adequacy of the model are discussed. Some related methods such as weighted regression and nonlinear models are also considered. A discussion of the general linear model is also included.

Appendix A gives some basic SAS programs and outputs for some of the analyses discussed in the body of the report. Appendix $B$ is a review of some of the matrix theoretic results which are useful in the development of linear models. 


\section{FOREWORD}

The Office for Analysis and Evaluation of Operational Data (AEOD) of the U.S. Nuclear Regulatory Commission (NRC) is continually reviewing data from U.S. commercial nuclear power plants. Statistical analysis forms an important part of this work. Accordingly, the Reliability and Risk Analysis Branch of AEOD has asked the Idaho National Engineering Laboratory (INEL) to write a series of reports presenting the appropriate statistical tools for the kinds of data most commonly encountered. These reports are being written in parallel with a series of studies on the performance of safety systems in nuclear power plants, and they reflect the influence of those studies.

The reports are expected to provide tools and guidance to analysts of NRC data, although the statistical methods can, by their nature, be applied to data from many other fields. The reports are intended to quickly help a new user. The report on collecting operational data should be understandable by anyone with a technical background, although the examples are slanted towards an engineer with nuclear experience. The reports on statistical methods should be readable and immediately usable by a person with training in statistics but with no experience analyzing such data. Some of the reports, of necessity, are more advanced than others; for example, the report on loglinear modeling will be easier to understand if the reader has first assimilated the reports on binomial data, Poisson data, and linear models. Nevertheless, the reports are all intended to be introductory to the extent possible, suitable as brief self-study texts to help readers move quickly to the tasks of data collection and analysis. In addition, the reports should be usable as texts or references in short courses for persons with less training.

The first reports written or planned in this series are

Collecting Operational Event Data for Statistical Analysis, September 1994, EGG-RAAM-10086, by Corwin L. Atwood

Hits per Trial: Basic Analysis of Binomial Data, September 1994, EGGRAAM-11041, by Corwin L. Atwood

Events in Time: Basic Analysis of Poisson Data, September 1994, EGG-

RAAM-11088, by M. E. Engelhardt 
Modeling Patterns in Data Using Linear and Related Models, June 1996, INEL-95/0120, by M. E. Engelhardt

Modeling Patterns in Count Data Using Loglinear and Related Models, December 1995, INEL-95/0121, by Corwin L. Atwood

Statistical Analysis of Random Duration Times, April 1996, INEL95/0206, by M. E. Engelhardt

Practical Guidance for Statistical Analysis of Operational Event Data, October 1995, INEL-95/0234, by Corwin L. Atwood 


\section{ACKNOWLEDGMENTS}

Cory Atwood and Cindy Gentillon supplied the data used in the examples, and also provided extremely helpful review comments. Thanks are also due to Harry Martz for helpful review comments of an earlier draft. 


\section{CONTENTS}

ABSTRACT $\ldots \ldots \ldots \ldots \ldots \ldots \ldots \ldots \ldots \ldots \ldots \ldots \ldots \ldots \ldots \ldots \ldots$

SUMMARY $\ldots \ldots \ldots \ldots \ldots \ldots \ldots \ldots \ldots \ldots \ldots \ldots \ldots \ldots \ldots \ldots \ldots \ldots$

FOREWORD $\ldots \ldots \ldots \ldots \ldots \ldots \ldots \ldots \ldots \ldots \ldots \ldots \ldots \ldots \ldots \ldots \ldots$ vii

ACKNOWLEDGMENTS $\ldots \ldots \ldots \ldots \ldots \ldots \ldots \ldots \ldots \ldots \ldots \ldots \ldots \ldots \ldots$

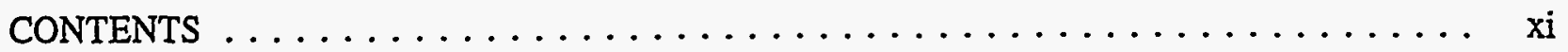

INTRODUCTION $\ldots \ldots \ldots \ldots \ldots \ldots \ldots \ldots \ldots \ldots \ldots \ldots \ldots \ldots \ldots \ldots$

REGRESSION MODELS $\ldots \ldots \ldots \ldots \ldots \ldots \ldots \ldots \ldots \ldots \ldots \ldots \ldots$

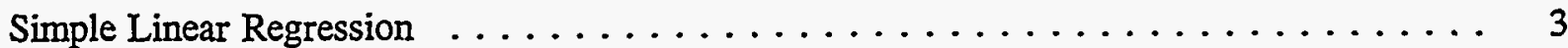

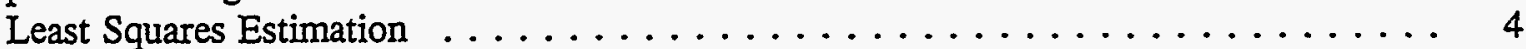

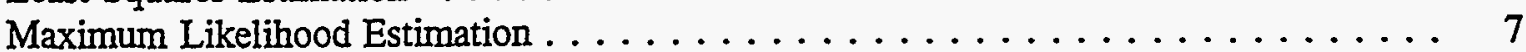

Diagnostic Procedures . . . . . . . . . . . . . . . . . . . . 8

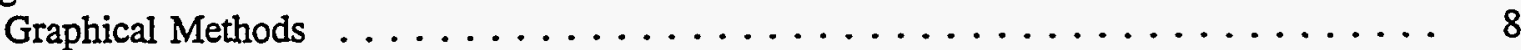

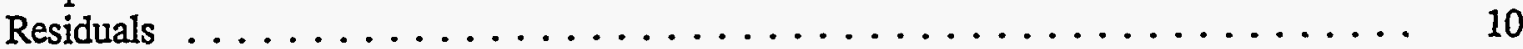

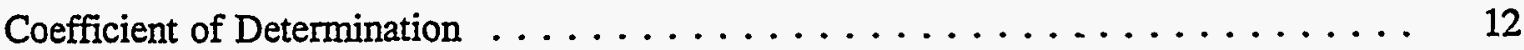

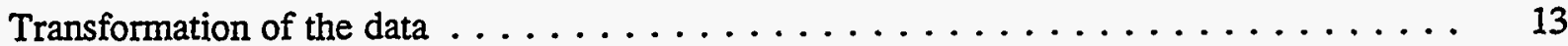

Nonlinear Least Squares $\ldots \ldots \ldots \ldots \ldots \ldots \ldots \ldots \ldots \ldots \ldots$

Weighted Least Squares $\ldots \ldots \ldots \ldots \ldots \ldots \ldots \ldots \ldots \ldots \ldots \ldots$

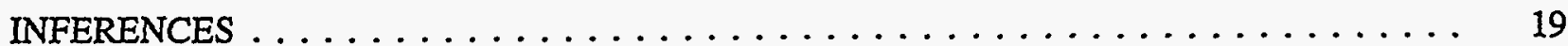

Distributional Properties . . . . . . . . . . . . . . . . . . . 19

Confidence Limits and Tests . . . . . . . . . . . . . . . . . . . . . . 19

Confidence Limits . . . . . . . . . . . . . . . . . . . . . . . . . . 19

Tests of Hypotheses . . . . . . . . . . . . . . . . . . . . . 20

Testing for a Trend . . . . . . . . . . . . . . . . . . . . . 21

Confidence Limits for the Mean Response . . . . . . . . . . . . . . . 23

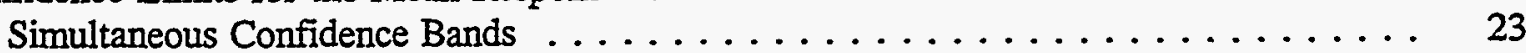

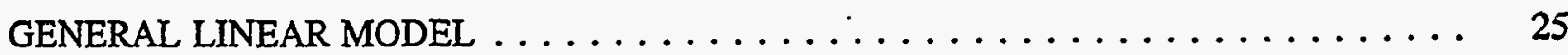

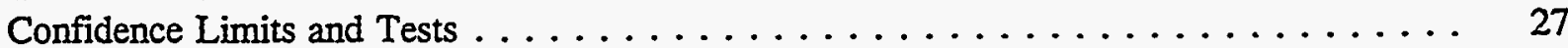

Properties of the Estimators . . . . . . . . . . . . . . . . . . . 28

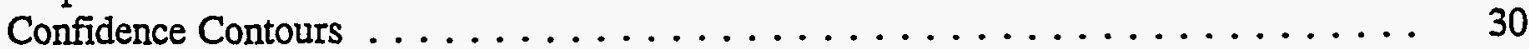

Testing Parts of a Model . . . . . . . . . . . . . . . . . . . . . 33

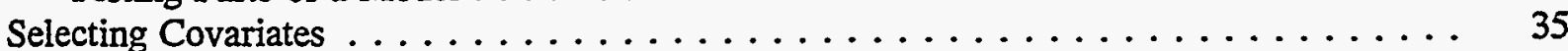

Stepwise Model Selection . . . . . . . . . . . . . . . . . 35

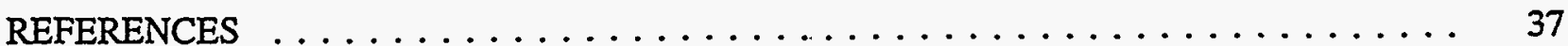


Apperidix A: Statistical Computation $\ldots \ldots \ldots \ldots \ldots \ldots \ldots \ldots \ldots \ldots \ldots \ldots$

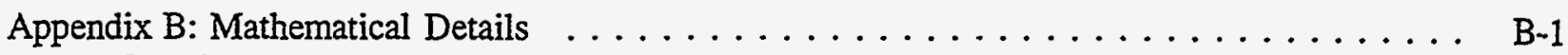

Matrix Notation . . . . . . . . . . . . . . . . . . .

Formulation of the Model $\ldots \ldots \ldots \ldots \ldots \ldots \ldots \ldots \ldots \ldots \ldots$

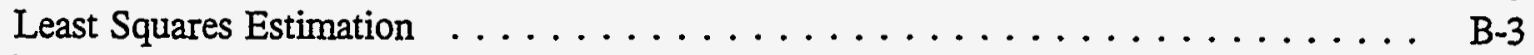

Properties . ............................

Maximum Likelihood $\ldots \ldots \ldots \ldots \ldots \ldots \ldots \ldots \ldots \ldots \ldots \ldots \ldots$

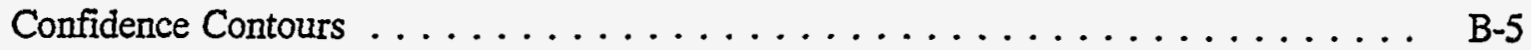

Correlated Errors $\ldots \ldots \ldots \ldots \ldots \ldots \ldots \ldots \ldots \ldots \ldots \ldots$ B-5

\section{FIGURES}

Figure 1. Plot of EDG failure rate versus age of plant. $\ldots \ldots \ldots \ldots \ldots$

Figure 2. Plot of EDG failure rate versus plant age, with linear and exponential curves. . . . . . 9

Figure 3. Residual plot of observed response with linear part removed. . . . . . . . . . . 10

Figure 4. Log-failure rate versus plant age for EDG data. . . . . . . . . . . . . . . . . 14

Figure 5. Residual plot for EDG log-failure rate versus plant age. . . . . . . . . . . . 15

Figure 6. Normal probability plot of standard normal quantiles versus standardized residuals. . 16

Figure 7. Plot of normalized radiation exposure versus year. . . . . . . . . . . . 22

Figure 8. Point-wise limits and simultaneous confidence band for a linear regression. . . . . . 24

Figure 9. Point-wise limits and simultaneous confidence band for exponential regression. . . . 25

Figure $10.95 \%$ confidence contour for regression coefficients. . . . . . . . . . 33

\section{TABLES}

Table 1. Radiation exposure (rem), plant years and rate (rem/year), 1987-95. . . . . . . 22

\section{EXAMPLES}

Example 1. Failure rate of emergency diesel generators versus plant age. . . . . . . . . 2

Example 2. Radiation exposure for nuclear plants in the U. S. for the period 1987-1995. . . . . 2 


\section{Modeling Patterns in Data Using Linear and Related Models}

\section{INTRODUCTION}

This report is about linear models and their application to operational data. It is often the case with a study that the value of an observation of interest is dependent on one or more other related quantities. The observation of interest will be called the response variable, and the related quantities which affect the response variable will be called covariates. Another term often used for the response variable is the dependent variable, and other terms often used for covariates are independent variables or explanatory variables. Throughout this report, an observation of the response variable will be denoted by $y$, and covariates will be denoted by $x$. In some cases, there will be more than one covariate, and in such cases, the same notation will be used, but with subscripts; $x_{1}, x_{2}$, etc.

Typically, observed values of a response variable will tend to vary from one observation to the next, and measured responses $y$ are modeled as realizations of a random variable $Y$. On the other hand, covariates are often precisely known, in which case they are assumed to be nonrandom variables. It is convenient to write the model as the sum of two terms, one a nonrandom (deterministic) part and the other a random error. Specifically, the general form is

$Y=$ nonrandom part + random error.

Unless otherwise indicated, we will assume throughout this report that the random error has mean value zero and constant variance. In other words, $E(Y)=$ nonrandom part, and $\operatorname{var}(Y)=$ constant. The mean, $E(Y)$, is a function of the covariates, whose values are known, and various parameters whose values are unknown. An important first analysis is to estimate from data the values of these unknown parameters. In order to do this, it is necessary for the analyst to make assumptions about the form of the nonrandom part. Such assumptions should be checked by the analyst, and diagnostic procedures for doing this will be discussed later in the report. We will first consider two examples which are based on operational data. 


\section{EXAMPLES}

The following examples will be used to illustrate the modeling and analysis of data such as that discussed above.

Example 1. Failure rate of emergency diesel generators versus plant age.

It is often of interest to study whether the reliability of some type of system is getting better (or worse) as the system ages. In this example, the response variable is an estimate of the failure rate for emergency diesel generators (EDGs) for each power plant in a recent study of EDG performance. The covariate in this example is plant age. The age of each plant in the study is measured from its low power license date. The unit of measure for the response variable is failures per plant year.

For a second example, we consider the following:

Example 2. Radiation exposure for nuclear plants in the U. S. for the period 1987-1995.

We consider radiation exposure data for the U. S. nuclear industry. These data include the annual radiation exposure (in rem) per plant and the operating time (in plant years) for each U.S. nuclear plant for each of the years 1987 through 1995. It would be of interest to see if there is a trend with respect to time. For example, a decreasing trend might be the expected result of an ongoing industry-wide safety program.

This example involves three numerical variables: the calendar year, the annual radiation exposure, and the annual operating time. Calendar year is the covariate of primary interest in this example. This is because the question of interest is the possible existence of a trend with respect to calendar year. Because the plant operating time is not constant from year to year, it must be taken into account in some way. One possibility would be to divide each plant's annual radiation measurements (exposure) by the corresponding operating time (exposure time), so that exposure levels are comparable. We then, treat this "normalized" annual radiation measurement as the response variable, and the calendar year as the covariate. In other words, the total amount of time that the plants are in operation will vary from one calendar year to the next, and the annual radiation exposure should be normalized accordingly. The response variable in this example is the normed exposure $y=$ exposure/(exposure time), and the covariate is $x=$ year. A second possibility would be to treat both exposure time and calendar year as two separate covariates. Later, we will treat this example both ways and compare the results.

A model is called a linear model if the nonrandom part is a linear function of the unknown 
parameters. For example, a model with two covariates and three unknown parameters $\beta_{0}, \beta_{1}$ and $\beta_{2}$ such that $E(Y)=\beta_{0}+\beta_{1} x_{1}+\beta_{2} x_{2}$ is a linear model. For another example, suppose there is only one covariate $x$, and three unknown parameters $\beta_{0}, \beta_{1}$ and $\beta_{2}$, with $E(Y)=\beta_{0}+\beta_{1} x+\beta_{2} x^{2}$. This is also a linear model since it is linear in the parameters, and it also illustrates that the deterministic part need not be a linear function of covariates. Consider a third example, involving a model with one covariate $x$, three unknown parameters $\beta_{0}, \beta_{1}$ and $\beta_{2}$, but $E(Y)=\beta_{0}+\beta_{1} \exp \left(\beta_{2} x\right)$. This is a linear function of the parameters $\beta_{0}$ and $\beta_{1}$, but not of $\beta_{2}$. Consequently this is not a linear model.

Applications of linear models generally fall into two special cases: regression analysis and analysis of variance (ANOVA). In regression applications, covariates are variables which are measured on a numerical scale, such as time or temperature. Typically with ANOVA, covariates are on a "nominal scale" with no inherent numerical structure. However, for convenience we usually set up dummy variables with indicator variables ( 0 or 1$)$ as the covariates.

This report will emphasize regression analysis without further discussion of ANOVA. Some diagnostic procedures for checking the model assumptions will also be discussed, and the methods will be illustrated by application to real data.

\section{REGRESSION MODELS}

The term regression was used by F. Galton (1889), a nineteenth century scientist, to describe a phenomenon involving heights of fathers and their sons. Specifically, the study considered paired data, $\left(x_{i}, y_{i}\right)$, where $x_{i}$ and $y_{i}$ represent, respectively, the heights of the $i$ th father and his son. One result of this work was the derivation of a linear relationship $y=a+b x$ for use in predicting a son's height given the father's height. It was observed that if a father was taller than average, the son tended to also be taller than average, but not by as much as the father. Similarly, sons of fathers who were shorter than average tended to be shorter than average, but not by as much as the father. This effect, which is known as "regression toward the mean", provides the origin of the term regression analysis, although the method is generally applicable to a wide variety of problems. This example illustrates an important special case, usually called the simple linear regression model and the next section deals with this case.

\section{Simple Linear Regression}

Consider a model of the form

$$
Y=\beta_{0}+\beta_{1} x+e
$$


where $e$ is a random error term with mean zero and variance independent of $x$. This model is called the simple linear model. In this section we derive estimates of the parameters $\beta_{0}$ and $\beta_{1}$ of Model (1) using a standard approach.

\section{Least Squares Estimation}

Suppose $x_{1}, x_{2}, \ldots, x_{\mathrm{n}}$ are known, nonrandom covariate values, and that there is an observation of the random response variable corresponding to each of these values, yielding observed values $y_{1}, y_{2}$, $\ldots, y_{\mathrm{n}}$. The ideal situation for fitting a straight line would be for all the pairs $\left(x_{\mathrm{i}}, y_{\mathrm{i}}\right)$ to fall on a straight line in which case a linear function could be determined algebraically. This is unlikely to happen in practice because the $y_{\mathrm{i}} \mathrm{s}$ are observed values of random variables. In order to check the assumption of linearity, there exist useful procedures such as a scatter diagram, which is simply a plot of the data in an $x-y$ coordinate system. Such diagrams will be discussed later in a section on diagnostics. The ith observed value of the response variable can be related to the Model (1), at least conceptually, as $y_{i}=\beta_{0}$ $+\beta_{1} x_{i}+e_{i}$. The term $e_{i}$ is the difference between what is actually observed on the $i$ th trial and the theoretical expected value $E\left(Y_{i}\right)=\beta_{0}+\beta_{1} x_{i}$. Notice that the $e_{i} s$ cannot be computed because the parameter values $\beta_{0}$ and $\beta_{1}$ are unknown and must be estimated from the data. If the analyst decides that a linear relationship is appropriate, the next step is to fit a straight line through the points in such a way as to minimize, in some sense, the resulting observed deviations of the $y_{\mathrm{i}}$ s from the fitted line.

Different criteria for such a fit lead to different functions of the $e_{i} \mathrm{~s}$, but we will use a standard approach called the method of Least Squares (LS) which involves minimizing the sum of the squared deviations from the fitted line. That is, we seek values of $\beta_{0}$ and $\beta_{1}$, say $\hat{\beta}_{0}$ and $\hat{\beta}_{1}$, that minimize the sum of squares of the $e_{\mathrm{i}} \mathrm{s}$,

$S=\Sigma e_{\mathrm{i}}^{2}=\Sigma\left[y_{\mathrm{i}}-\beta_{0}-\beta_{1} x_{\mathrm{i}}\right]^{2}$

A familiar technique from differential calculus can be used to minimize $S$. This involves taking derivatives of $S$ with respect to both $\beta_{0}$ and $\beta_{1}$, and setting the derivatives equal to zero. The LS estimates are obtained as solutions to the following pair of linear equations in the unknown parameters $\beta_{0}$ and $\beta_{1}$ (usually called the normal equations):

$$
\begin{aligned}
\left(\Sigma x_{i}\right) \beta_{0}+\left(\Sigma x_{i}^{2}\right) \beta_{1} & =\Sigma x_{i} y_{i} \\
n \beta_{0}+\left(\Sigma x_{i}\right) \beta_{1} & =\Sigma y_{i}
\end{aligned}
$$

These equations can be solved simultaneously with standard methods for solving systems of linear equations, giving explicit LS estimates 


$$
\begin{aligned}
\hat{\beta}_{1} & =\left[\Sigma x_{i} y_{i}-\left(\Sigma x_{i}\right)\left(\Sigma y_{i}\right)\right] /\left[\Sigma x_{i}^{2}-\left(\Sigma x_{i}\right)^{2}\right] \\
& =\Sigma\left(x_{i}-\bar{x}\right) y_{i} / \Sigma\left(x_{i}-\bar{x}\right)^{2} \\
\hat{\beta}_{0} & =\bar{y}-\hat{\beta}_{1} \bar{x}
\end{aligned}
$$

with $\bar{x}=\Sigma x_{i} / n$ and $\bar{y}=\Sigma y_{i} / n$. Although these estimates are fairly easy to compute with a hand-held calculator, is it common in practice to use a computer and statistical software. Both Examples 1 and 2 have been analyzed using the SAS procedure PROC REG. An example program is given in Appendix $A$, and the numerical results are discussed in a later section. First, we discuss a few properties of the LS estimators. There are many other statistical software packages available for this purpose, e.g., JMP, S-Plus and Stata.

The results thus far have dealt with fitting a straight line to a set of data points, and not much has been required in the way of mathematical assumptions. In the discussion which follows, assumptions will be considered which guarantee that the LS estimates have desirable properties. We will start with the following set of assumptions about the errors in Model (1):

\section{Assumptions}

$$
\begin{aligned}
& \text { 1. The mean of an error is zero, } \mathrm{E}(e)=0 \text {. } \\
& \text { 2. Variances of errors are constant, } \operatorname{var}(e)=\sigma^{2} \text {. } \\
& \text { 3. Errors are statistically independent. } \\
& \text { 4. Errors are normally distributed, } \mathrm{N}\left(0, \sigma^{2}\right) \text {. }
\end{aligned}
$$

The first assumption is appropriate when there is no bias in what we observe. This would happen, for example, if measuring devices have been properly calibrated, and there is no other uncontrolled effect causing the average measurement to be on one side (either too high or too low) of the true value. More explicitly, if a balance scale has been properly calibrated, the mean variation in repeated measurements will be zero. However, if the balance scale has been dropped, or subjected to some other shock, further measurements might tend to be consistently too high (or too low) depending on the circumstances. The second assumption means that the magnitude of the error (either positive or negative) tends to be the same, regardless of the value of the covariate. When the variance is constant, the model is called homoscedastic, and otherwise it is called heteroscedastic. The third assumption means 
that the value of one error does not effect the value of any of the other errors. In other words, if a particular error has a large magnitude, this does not affect the likelihood of one or more other errors also being large. Similarly, a small error does not mean that other errors will be small. The fourth assumption says that the errors will have a probability distribution that is symmetric and bell-shaped. This distribution, which is called normal or Gaussian, is appropriate with many random quantities which occur in nature.

The LS estimators are linear functions of the response variables $Y_{1}, Y_{2}, \ldots, Y_{n}$, which makes it fairly easy to derive their expected values and variances. It can be shown that under the above set of assumptions the LS estimators are unbiased, in the usual sense that the expected value of the estimator is the parameter value it is intended to estimate. Furthermore, it can be shown that within the class of all linear unbiased estimators, the LS estimators have minimum variance. Thus, the LS estimators are often referred to as Best Linear Unbiased Estimators (BLUEs). Under Assumptions 1 through 3, the IS estimators have the following properties:

1. $E\left(\hat{\beta}_{0}\right)=\beta_{0}, \operatorname{var}\left(\hat{\beta}_{0}\right)=\sigma^{2} \Sigma x_{i}^{2} /\left[n \Sigma\left(x_{i}-\bar{x}\right)^{2}\right]$

2. $E\left(\hat{\beta}_{1}\right)=\beta_{1}, \operatorname{var}\left(\hat{\beta}_{1}\right)=\sigma^{2} / \Sigma\left(x_{i}-\bar{x}\right)^{2}$,

3. $\operatorname{cov}\left(\hat{\beta}_{0}, \hat{\beta}_{1}\right)=-\sigma^{2} \bar{x} / \Sigma\left(x_{i}-\bar{x}\right)^{2}$.

4. If $a$ and $b$ are real constants, then $\mathrm{E}\left(a \hat{\beta}_{0}+b \hat{\beta}_{1}\right)=a \beta_{0}+b \beta_{1}$ for all values of $\beta_{0}$ and $\beta_{1}$,

5. $a \hat{\beta}_{0}+b \hat{\beta}_{1}$ is the BLUE of $a \beta_{0}+b \beta_{1}$.

The last property is useful because many important quantities can be written as linear combinations of the regression parameters. For example, if $x$ is a known value, a predicted value of the response variable,

$\hat{y}=\hat{\beta}_{0}+\hat{\beta}_{1} x$

is a linear combination of the estimators. According to property 5, this is the BLUE of the mean response $E(Y)=\beta_{0}+\beta_{1} x$.

It should be noted that all of the properties of moments stated above can be derived under a weaker set of assumptions. In particular, the above properties about BLUEs hold under Assumptions 1 and 2, and instead of Assumption 3, assuming that errors are pair-wise uncorrelated, $\operatorname{cov}\left(e_{\mathrm{i}}, e_{\mathrm{j}}\right)=0$ 
whenever $i \neq j$. This result was first noted by C. F. Gauss (1823), and in the more recent literature, these results have been restated in terms of matrices (see e.g. Bain and Engelhardt, 1992, p. 517).

The IS method does not directly provide an estimate of $\sigma^{2}$, but it can be shown that an estimate of $\sigma^{2}$ which is unbiased in the usual sense, $E\left(s^{2}\right)=\sigma^{2}$, is given by

$s^{2}=\Sigma\left[y_{i}-\hat{\beta}_{0}-\hat{\beta}_{1} x_{i}\right]^{2} /(n-2)$.

\section{Maximum Likelihood Estimation}

Another often used approach to estimation is based on the Principle of Maximum Likelihood (ML). This approach makes use of the assumptions of errors which are independent and normal (Assumptions 3 and 4). The joint probability density function (pdf) of the observed $y_{i}$ s is thus the product of $n$ normal probability density functions whose means are $\beta_{0}+\beta_{1} x_{\mathrm{i}}$ and with the same variance $\sigma^{2}$. As a function of the parameters for a fixed set of data values $y_{\mathrm{i}}$, this product is called the likelihood function. Specifically, we have

$$
\begin{aligned}
L & =L\left(\beta_{0}, \beta_{1}, \sigma^{2}\right) \\
& =\Pi\left(2 \pi \sigma^{2}\right)^{-1 / 2} \exp \left[-\left(y_{i}-\beta_{0}-\beta_{1} x_{i}\right)^{2} /\left(2 \sigma^{2}\right)\right] \\
& =\left(2 \pi \sigma^{2}\right)^{-\pi / 2} \exp \left[-\Sigma\left(y_{i}-\beta_{0}-\beta_{1} x_{i}\right)^{2} /\left(2 \sigma^{2}\right)\right] .
\end{aligned}
$$

Given the data, MI estimates are the parameter values for which the likelihood function is a maximum. Thus, the application of the ML principle to regression involves taking partial derivatives of the loglikelihood function $\ln L$, setting these partial derivatives equal to zero, and then solving the resulting set of equations for the estimates. The ML estimates of $\beta_{0}$ and $\beta_{1}$ are identical to the LS estimates. It is also possible to derive an ML estimate $\hat{\sigma}^{2}$ of $\sigma^{2}$, and it is closely related to the estimate $s^{2}$ stated above. Specifically, the ML estimate $\hat{\sigma}^{2}$ is $(n-2) s^{2} / n$; i.e., the same numerator as for $s^{2}$, but with $n-2$ in the denominator replaced by $n$. The $\mathrm{ML}$ is generally not used as a point estimate in practice, primarily because it is a biased estimate of $\sigma^{2}$. The ML estimate tends to underestimate $\sigma^{2}$, although for large $n$, $s^{2}$ and the MLE of $\sigma^{2}$ are close, and the bias of the MLE is negligible. We will use the unbiased estimate discussed above and continue to denote it by $s^{2}$. Because the LS and ML estimates of the coefficients are identical under the stated assumptions, we will simply refer to either of these as the IS estimates. 


\section{Diagnostic Procedures}

As mentioned previously, it is desirable to have diagnostic procedures which can be used to check model assumptions such as the linearity of the nonrandom part of Model (1).

\section{Graphical Methods}

Even if Model (1) is correct, we would not expect the observed points to all line on the same straight line due to random variation. However, we would expect most of the points to cluster about a line when plotted on $x-y$ coordinates. A plot of paired values $\left(x_{i}, y_{i}\right)$ on $x-y$ coordinates is called a scatter diagram. To illustrate this basic method, we consider data for Example 1, involving EDG failure rates for $n=109$ plants. The resulting scatter diagram is shown in Figure 1.

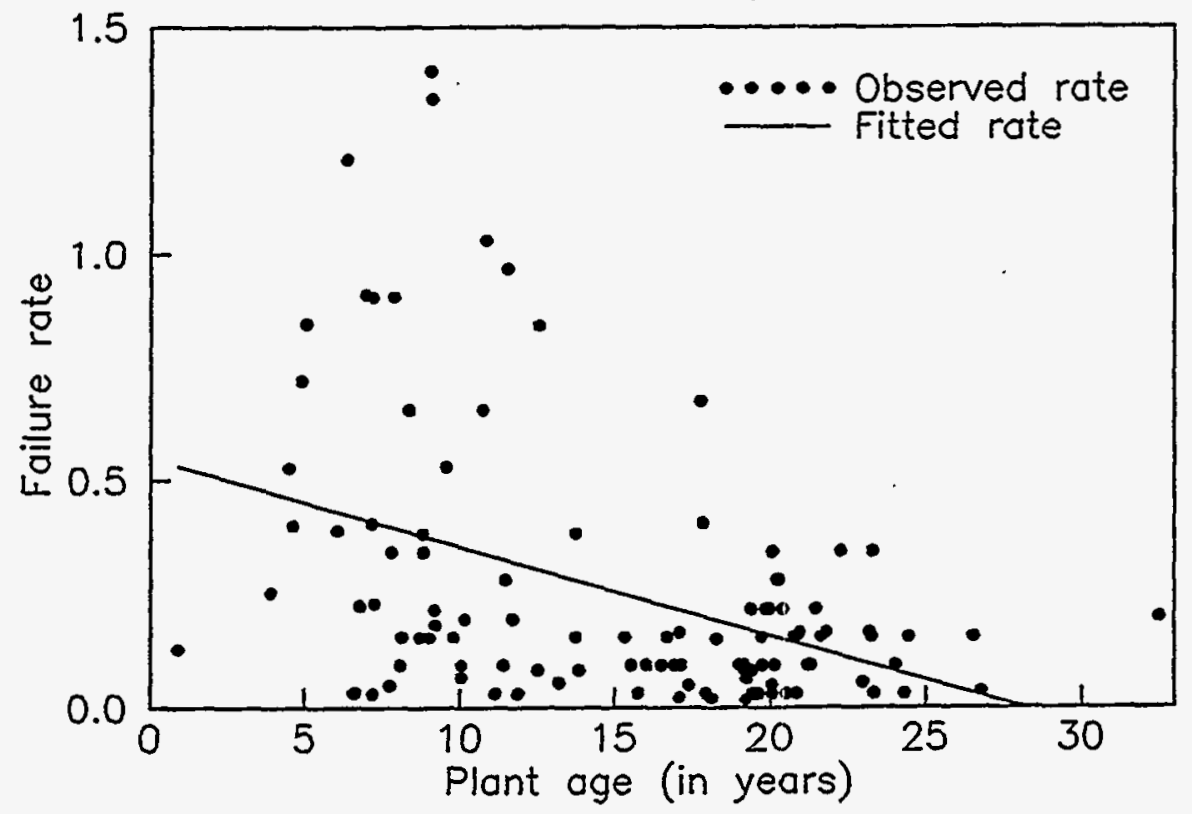

Figure 1. Plot of EDG failure rate versus age of plant.

The estimates of $\beta_{0}$ and $\beta_{1}$, based on the output from SAS are $\hat{\beta}_{0}=0.5486$ and $\hat{\beta}_{1}=-0.0195$, and the fitted linear equation is $\hat{y}=0.5486-0.0195 x$. The graph of this linear function has also been included for comparison in Figure 1. It should be noted that the there is a discernable pattern of 
skewness in the scatter diagram. We note, in particular, that $62 \%$ of the $y_{i}$-values lie below the line, while only $38 \%$ lie above the line. If, in fact, the nonrandom part of the model is linear and the errors are normal, we should expect to see closer to $50 \%$ both above and below the line. Finally, if the fitted line is to be used as an estimate or predictor of failure rate, then it will not be useful over the range of the covariate where the line is negative (roughly plants older than 28 years).

An obvious alternative analysis would consider some other monotonic increasing function for the nonrandom part, such as an exponential function. In Figure 2, we see the graph (the dashed curve) of a fitted exponential curve. For the moment we will ignore the details regarding how such a curve is fitted to the data. This will be considered later. There is a noticeable pattern in the plot. In particular, there

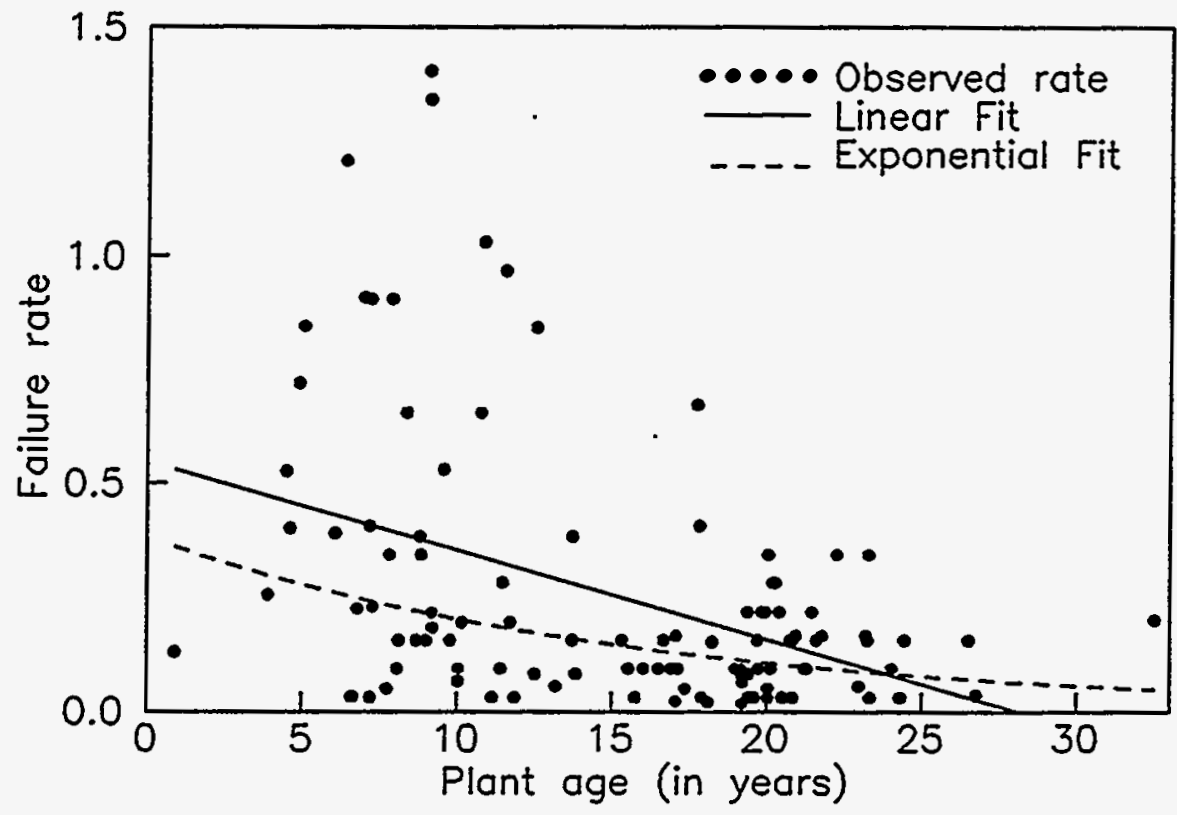

Figure 2. Plot of EDG failure rate versus plant age, with linear and exponential curves.

is more vertical scatter to the points for smaller values of the covariate. This would suggest a problem with the assumption of constant variance. Note that it can be difficult to simultaneously spot both a nonlinearity and nonconstant variance in the same plot. 


\section{Residuals}

Another type of type of diagnostic procedure, called a residual analysis can also be used. The quantity

$\hat{y}_{\mathrm{i}}=\hat{\beta}_{0}+\hat{\beta}_{1} x_{\mathrm{i}}$

is an estimated or "predicted" value of the mean response, $\mathrm{E}\left(Y_{\mathcal{i}}\right)=\beta_{0}+\beta_{1} x_{\mathrm{i}}$, and the differences

$\hat{e_{i}}=y_{i}-\hat{y}_{i}$

are called residuals. A residual plot enables the analyst to inspect the data for the presence or absence of a pattern in the variability with variation due to the nonrandom part of the model removed. Figure 3 gives a residual plot for Model (1). Given some other fitted model, such as the exponential model, a similar analysis could also be done for that model.

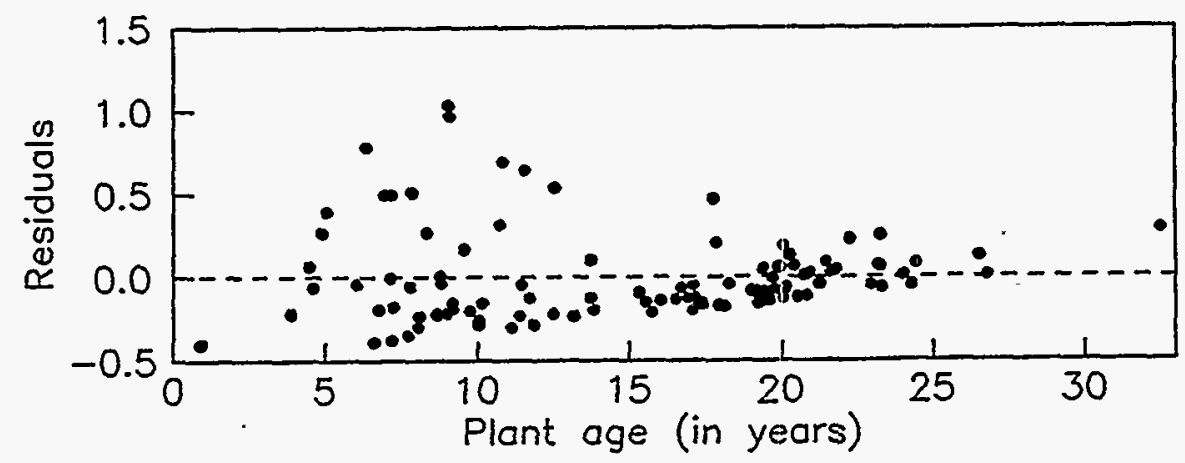

Figure 3. Residual plot of observed response with linear part removed.

There is a noticeably larger amount of vertical scatter for smaller values of the covariate, particularly for plants with ages less than 15 years. It can be seen from Figure 2, that such a pattern would also be present in a residual plot with the exponential fit, but to a lesser degree. In a later section, we will discuss transformation methods which can sometimes take care of problems due to both lack of fit and the lack of constant variance.

Another variation on the procedure with residuals is to plot standardized residuals. The standardized residuals are sometimes called studentized residuals, although this terminology is not 
standard in the literature. A difficulty with ordinary residuals, as described above, is that they do not all have the same variance. In particular, the variance of the $i$ th residual is $\operatorname{var}\left(\hat{e}_{\mathrm{i}}\right)=\left(c_{\mathrm{i}} \sigma\right)^{2}$ where

$c_{\mathbf{i}}=\left[1-1 / n-\left(x_{\mathrm{i}}-\bar{x}\right)^{2} / \Sigma\left(x_{\mathrm{j}}-\bar{x}\right)^{2}\right]^{1 / 2}$.

In order to adjust for this, one could divide each residual by the estimate of its standard deviation, thus obtaining the standardized residuals, $e_{i}^{*}=\hat{e}_{i} /\left(c_{i} s\right)$. If the assumptions about the random error hold, these standardized residuals have an approximately standard normal distribution when $n$ is large. This approximation can be useful when trying to decide whether one or more of them is larger than would be expected. Although the studentized residuals can be plotted as an option with SAS, we will not illustrate this here.

The residual plot shown in Figure 3 presents the residuals $\hat{e}_{i}$ on the vertical axis versus the covariate $x_{\mathrm{i}}$ on the horizontal axis. Some other related procedures which are sometimes helpful in spotting patterns in the residuals are to plot:

- $\hat{e}_{\mathrm{i}}$ (or $e_{\mathrm{i}}^{*}$ ) on the vertical axis versus $\hat{y}_{\mathrm{i}}$ on the horizontal axis,

- $\hat{y}_{i}$ on the vertical axis versus $y_{i}$ on the horizontal axis, and

- a normal probability plot (or a Q-Q plot) of the standardized residuals, $e_{\mathrm{i}}^{*}$.

This last procedure is a check on the normality assumption. As previously mentioned, if the assumptions about the random errors hold, then for large $n$ the standardized residuals are approximately standard normal, i.e., $N(0,1)$. This suggests using standard goodness-of-fit procedures available for data from random samples as a diagnostic tool for regression analysis. It is not quite correct to say that the standardized residuals are distributed as a random sample from the standard normal distribution, since residuals are correlated with each other and $s$ is used instead of $\sigma$. However, the correlation between residuals is relatively small and $s$ is close to $\sigma$ when $n$ is large. This suggests that techniques such as normal probability plots or Q-Q plots can be useful in checking the assumption of normal errors. Formal statistical tests for goodness-of-fit, such as the Shapiro-Wilk test, are also available in most PC-based statistical software packages. For a discussion of such plots and tests, see D'Agostino and Stephens (1986). The residual analysis considered above cast some doubt on the assumption of constant variance, so we will wait to illustrate any goodness-of-fit procedures. We will now discuss another aspect of the modeling problem. 


\section{Coefficient of Determination}

The sum of squares of the residuals is called the error sum of squares, denoted by

SSE $=\Sigma\left(\gamma_{i}-\hat{y}_{i}\right)^{2}$

The error sum of squares is also related to two other sums of squares,

$\operatorname{SST}=\Sigma\left(y_{i}-\bar{y}\right)^{2}$

called the total sum of squares, and

$\operatorname{SSR}=\Sigma\left(\hat{y}_{i}-\grave{y}\right)^{2}$

called the regression sum of squares.

If the deterministic part of Model (1) is constant (i.e. $\beta_{1}=0$ ), then all variation in the response data can be attributed to random error, and this would be appropriately measured by the SST, which assesses the differences between the response data and the overall average $\bar{y}$. If, on the other hand, the mean response varies linearly with respect to the covariate, then some of the variation in the response data can be attributed to this dependence on the covariate. In this case, the SSR, which assesses the difference between LS predictions of response and the overall average, is a measure of the variation which is "explained" by the linear relationship. In other words, if the slope of the line is zero (or near zero), then differences between the LS predicted response and overall average response will tend to be small, and SSR will be small. However, if the slope is large, most of the differences will tend to be large, yielding a large value of SSR. In the latter case, the SSE, which assesses the differences between the response data and the LS predictions of response, provides a measure of the random error in the data. Thus, the SSE is a measure of the "unexplained" variation or that which is attributed primarily to random error in the observed responses.

It can also be shown that the total sum of squares is obtained by adding the error sum of squares and the regression sum of squares,

SST $=$ SSE + SSR

This relationship represents a partitioning of the SST, into the two components SSE and SSR. As noted above, the SSR measures the variation which can be explained by the linear model, and the SSE measures 
the variation which is either left unexplained or due to randomness.

This relationship can also be used to develop a measure of the goodness-of-fit of the simple linear model known as the coefficient of determination and defined as

$r^{2}=\operatorname{SSR} / S S T$

It follows from the fact that $0 \leq \mathrm{SSR}=\mathrm{SST}-\mathrm{SSE} \leq \mathrm{SST}$, that $0 \leq r^{2} \leq 1$. There are two important limiting cases: (1) When the data pairs are collinear and the line has nonzero slope, SSE $=0$ and $r^{2}=$ 1 , and (2) when all $\hat{y}_{i}=\bar{y}$, SSR $=0$ and $r^{2}=0$. More generally, because SSR measures variation which can be attributed to the linear effect on the response variable, and SSE is a measure of the random error, $r^{2}$ will be near 0 if most of the variation is due to randomness and near 1 if most of the variation is due to the linear effect. Consequently, $r^{2}$ is a useful tool in testing the strength of the fit of Model (1). For the EDG failure rate problem (Example 1), the SAS output gives the value $r^{2}=0.1767$. There is a statistical test which can be performed, based on $r^{2}$, and it can be used to test the fit of the model. Specifically, under the assumptions already discussed for Model (1), we have that

$F=(n-2) r^{2} /\left(1-r^{2}\right)$

follows a so-called "F distribution" with $\nu_{1}=2$ degrees of freedom for the numerator and $\nu_{2}=n-2$ degrees of freedom for the denominator when $\beta_{1}=0$ (i.e. zero slope). Thus, a test of size $\alpha$ of the adequacy of Model (1), is to reject the hypothesis that $\beta_{1}=0$ if $F \geq F_{\alpha, 2, n-2}$. One of the requirements of this test is constant variance, which has been put in doubt in Example 1 by the residual analysis. Thus, we will not attempt to perform this test at this point.

\section{Transformation of the data}

As noted previously, it is possible in some cases to use transformation methods to solve both the lack of fit and the lack of constant variance problems. This involves application of some function to the response variable, and sometimes also to the covariate. One of the most useful transformations is based on taking the logarithm of the response variable. In connection with this procedure, consider the following "multiplicative error" model, called the exponential model:

$U=A \exp (B x) e^{*}$

where $U$ is now the response variable, and $e^{\pi}$ is a multiplicative error which has the lognormal 
distribution with parameters 0 and $\sigma^{2}$. That is, $\log (e)$ is normal with mean 0 and variance $\sigma^{2}$. This is not a linear model, in the sense discussed previously, but it can be transformed into a model which is a linear model. In particular, if $Y=\log (U)$, then

$Y=\log (A)+B x+e$

which is linear in the parameters $\beta_{0}=\log (A)$ and $\beta_{1}=B$. Also, the random term, $e=\log (e)$, is normal with mean 0 and variance $\sigma^{2}$.

We will illustrate the use of this transformation with the data for Example 1. The SAS analysis on the logarithms of the estimated EDG failure rates yields $\beta_{0}=-0.9637$ and $\beta_{1}=-0.0637$. The scatter diagram, with a logarithmic vertical scale, is shown in Figure 4.

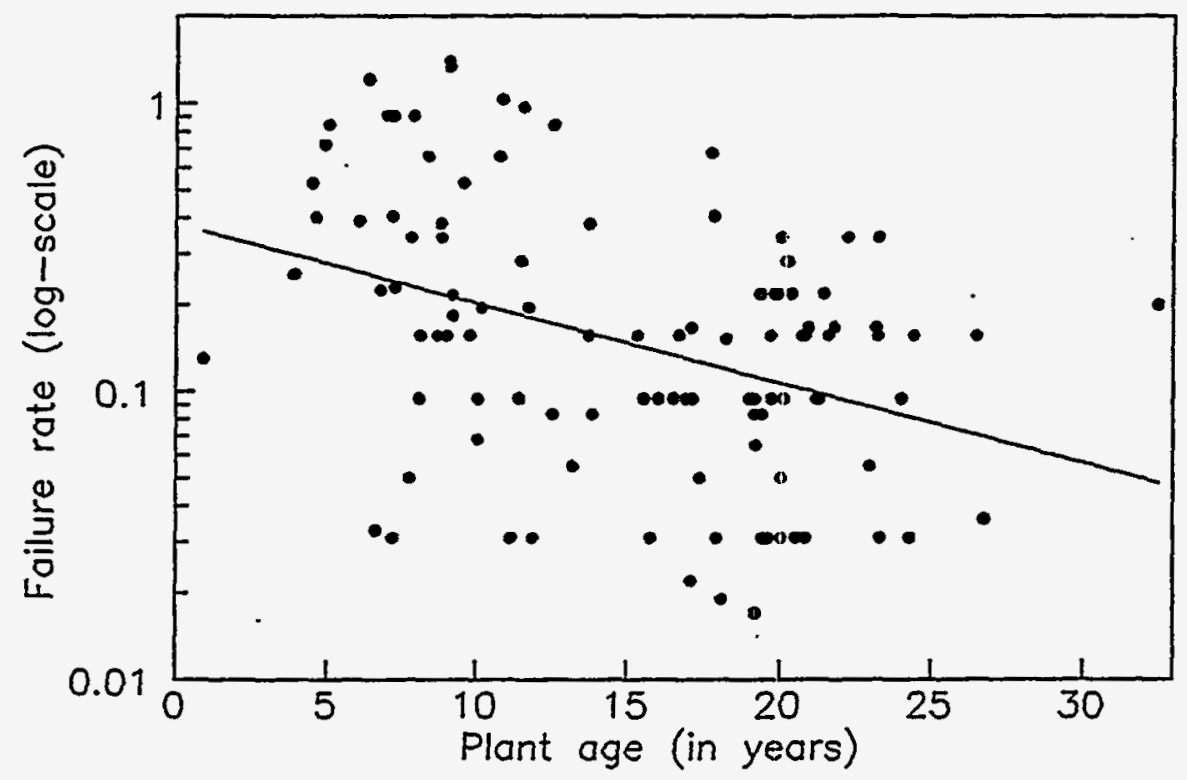

Figure 4. Log-failure rate versus plant age for EDG data.

It is clear from inspection of Figure 4 that the heteroscedasticity problem seen in Figures 1 and 2 is no longer present, or at least is not as pronounced. An assumption of a linear pattern in the log-data (corresponding to an exponential model without the transformation) is also quite plausible. Note in Figure 4, the vertical scale is a log-scale. Many graphics software packages have this as an option. 
Figure 5 shows the residuals for the log-data. This plot is consistent with the observation made above that an assumption of constant variance for the transformed data is plausible. The value of the

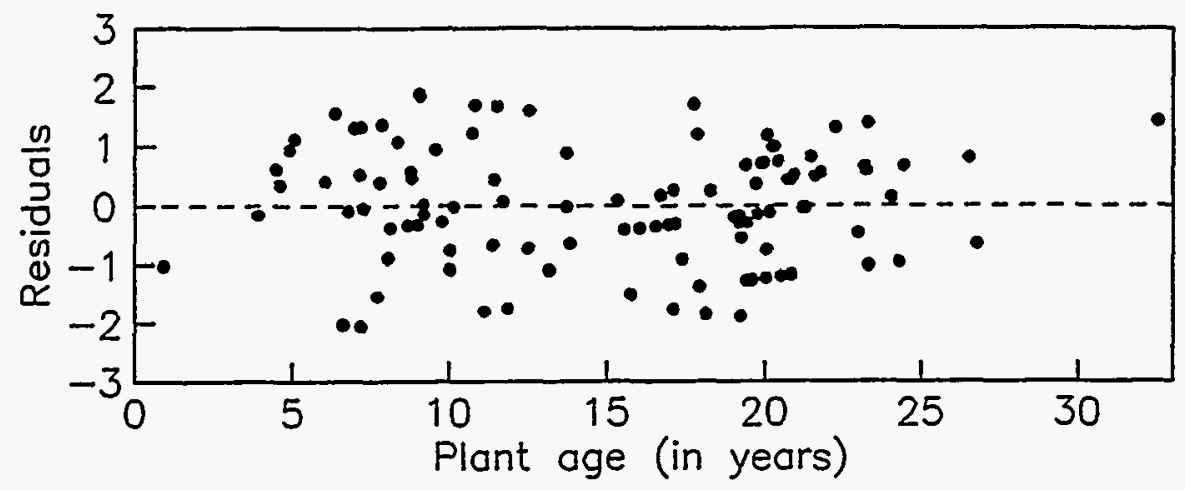

Figure 5. Residual plot for EDG log-failure rate versus plant age.

coefficient of determination for the log-transformed data is $r^{2}=0.1437$. The resulting F-statistic for testing this model is $F=17.95$, which has a $p$-value of 0.0001 . This is extremely strong evidence in favor of an approach which models dependence on plant age; e.g., the exponential model for failure rates or a simple linear model for the log-failure rates.

Another model called the power model is:

$\mathrm{W}=A t^{\mathrm{B}} e^{\mathrm{Z}}$

where $W$ is the response variable, $t$ is the covariate and $e^{*}$ is a multiplicative error which has the lognormal distribution with parameters 0 and $\sigma^{2}$. As in the case of Model (2), this is not a linear model, but it can be transformed into a model which is a linear model. In particular, if $Y=\log (W)$, then

$Y=\log (A)+B \log (t)+e$

which is linear in the parameters $\beta_{0}=\log (A)$ and $\beta_{1}=B$ with transformed covariate $x=\log (t)$. As with Model (2), the random term, $e=\log \left(e^{*}\right)$, is normal with mean 0 and variance $\sigma^{2}$. Many graphics software packages have an option of plotting with both horizontal and vertical log-scales.

As shown in Figures 4 and 5, the log-transformation of the data from Example 1 has the effect of reducing the nonlinearity as well as the heteroscedasticity. We will also check the normality assumption about the transformed errors $e_{\mathrm{i}}$, or equivalently, the lognormality of the multiplicative errors 
$e_{i}^{*}$ in Model (2). Figure 6 shows a plot of standardized residuals versus standard normal quantiles.

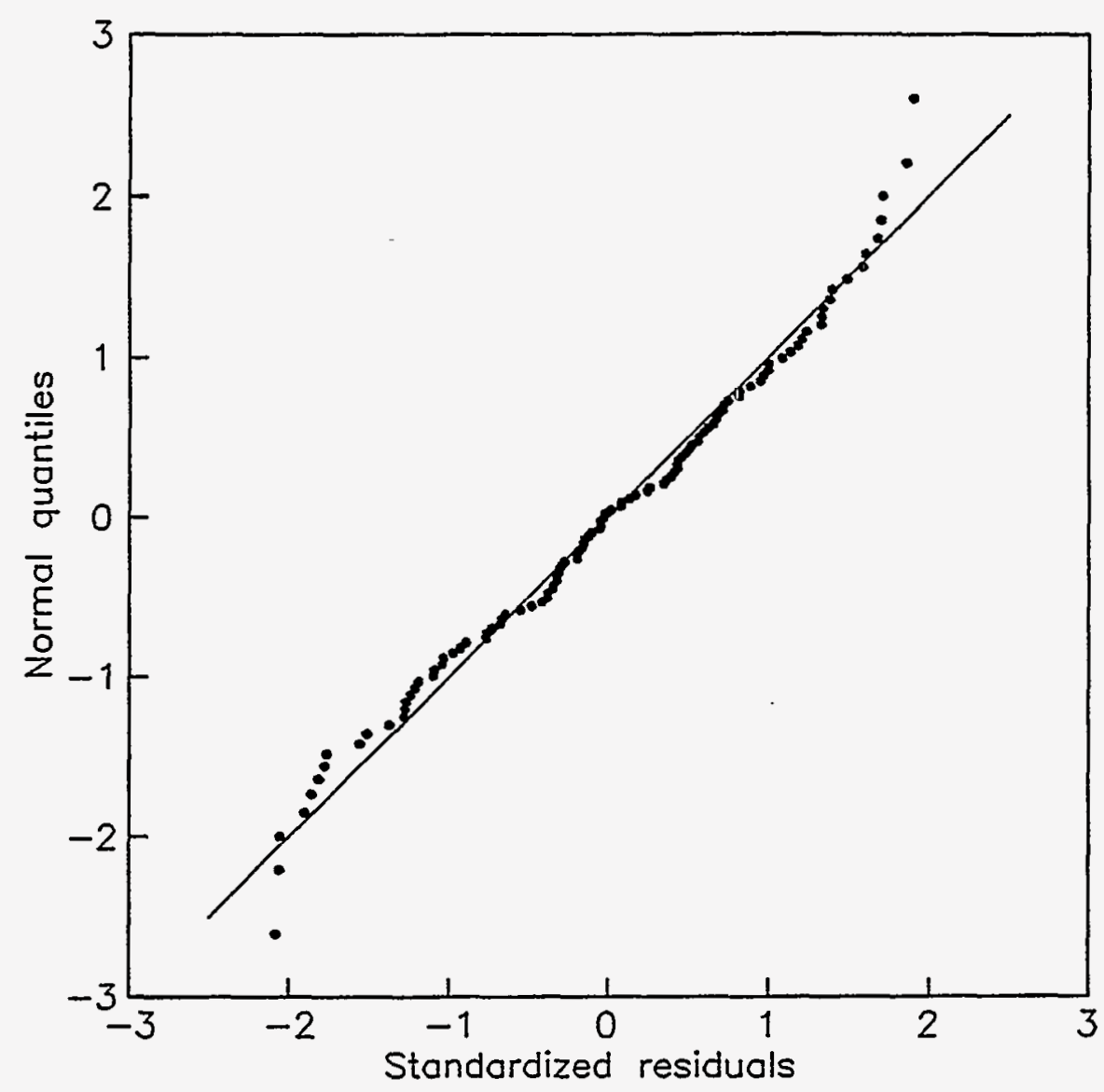

Figure 6. Normal probability plot of standard normal quantiles versus standardized residuals.

Inspection of Figure 6 shows most of the points lie fairly close to the diagonal line $(y=x)$. It is not uncommon with probability plots to see a few points which deviate from the line at one or both extremes. In the case of Figure 6 , a few points turn downward at the lower end and a few turn upward at the upper end of line. With normal probability plots, this situation is generally considered to be a symptom of a distribution which is symmetric but with thinner tails than a normal distribution (see D'Agostino and Stephens, 1986, p. 47). However, the assumption of normal errors appears to be good enough for practical purposes. 


\section{Nonlinear Least Squares}

When a model is nonlinear in the parameters, the entire process of estimation is radically different from the situation of the linear model. In the case of a single covariate $x$, instead of Model (1), we have a more general form

$Y=g(x ; \beta)+e$

where $\beta$ may represent a vector of parameters, $\beta_{0}, \beta_{1}$, etc., and $g$ is a function which is not linear in the $\beta_{i}$ S. The IS principle would say to find a vector, say $\hat{\beta}$, which would minimize the sum of squares

$S=\Sigma\left[y_{i}-g\left(x_{i} ; \beta\right)\right]^{2}$

Usually, when the function $g$ is not linear in the parameters, neither are the normal equations. Solutions of nonlinear equations are typically not available in closed form. If solutions exist they may not be unique, and generally they must be obtained by iterative numerical methods.

As we have discovered, some nonlinear models, such as Models (2) and (3) can be linearized by proper use of transformations. However, there are models which are potentially useful which cannot be transformed in this way. For example, consider the model

$Y=\beta_{0}+\beta_{1} \exp \left(\beta_{2} x\right)+e$

which has a vector of parameters $\beta=\left(\beta_{0}, \beta_{1}, \beta_{2}\right)$. This model was used in NUREG-1272, (1993, Section 2.1.9) to analyze trends in automatic reactor trips. The nonrandom part of the model is not linear in the parameter $\beta_{2}$, and there is no obvious way to transform it into a linear model. We will not pursue nonlinear models further, but there is a procedure in SAS called PROC NLIN which is capable of handling nonlinear regression problems. In addition, $J M P$ can also be used to fit a nonlinear model.

\section{Weighted Least Squares}

One of our basic assumptions with linear models is that the variance of the random errors is constant. It turns out that the LS approach, as already described, still provides unbiased estimators of the parameters when the variances are not constant, although they will no longer have the smallest variance in the class of linear functions of the response variables, $Y_{\mathrm{i}}$. There is a variation of the LS principle called Weighted Least Squares (WLS) principle which can be used to find the BLUEs in this 
more general setting. Suppose we associate a known positive "weight", $w_{\mathrm{i}}$, with the $i$ th observation, $y_{\mathrm{i}}$, where the $w_{i} s$ account for such things as differences in the variances of the $y_{i} s$. For the simply linear Model (1), the WLS approach amounts to solving for the values $\hat{\beta}_{0}$ and $\hat{\beta}_{1}$, that minimize the weighted sum of squares,

$S_{\mathrm{w}}=\Sigma w_{\mathrm{i}}\left[y_{\mathrm{i}}-\beta_{0}-\beta_{1} x_{\mathrm{i}}\right]^{2}$

The same approach used to solve the ordinary LS problem also works in this case, and the results are similar. This process involves taking derivatives of $S_{w}$ with respect to both $\beta_{0}$ and $\beta_{1}$, and setting the derivatives equal to zero. The WLS estimates are obtained as solutions to the following pair of normal equations:

$$
\begin{gathered}
\left(\Sigma w_{i} x_{i}\right) \beta_{0}+\left(\Sigma w_{i} x_{i}^{2}\right) \beta_{1}=\Sigma w_{i} x_{i} y_{i} \\
\left(w_{j}\right) \beta_{0}+\left(\Sigma w_{i} x_{i}\right) \beta_{1}=\Sigma w_{i} y_{i}
\end{gathered}
$$

These equations can be solved simultaneously with standard methods for solving systems of linear equations, giving explicit WLS estimates. As with the ordinary LS estimates, it is possible to solve for explicit solutions. An option in the SAS procedure PROC REG solves for the WLS estimates.

The WLS approach is more general than the LS approach and it can be used to derive BLUEs in cases where the constant variance property (Assumption 2) does not hold. In order to obtain the BLUEs, a special set of weights are required. In particular, suppose the variances of the responses have the form $\operatorname{var}\left(Y_{i}\right)=a_{i} \sigma^{2}$ where the $a_{i} s$ are known constants. In other words, the variances are not required to be the same for each response, but they are proportional to a known set of constants (the $a_{i} s$ ). The weights which are the reciprocals of these constants, $w_{\mathrm{i}}=1 / a_{\mathrm{i}}$, produce the BLUEs in this case. It should be noted that the unweighted estimators obtained using the LS method discussed previously provide unbiased estimators of the parameters in this more general situation; however, when the variances are not constant, the weighted estimators will have smaller variance. These results are discussed by Bain and Engelhardt (1992, Ch. 15).

There is one more aspect to weighted least squares, namely it can also be used with correlated responses. Suppose the observed responses are correlated and the covariances are known, except for an unknown scale factor. In particular, suppose for each $i$ and $j$,

$\operatorname{cov}\left(Y_{\mathrm{i}}, Y_{\mathrm{j}}\right)=\sigma^{2} a_{\mathrm{ij}}$

where each $a_{\mathrm{ij}}$ is a known constant. Notice if $i=j$, the variance is a special type of covariance and 
instead of the single subscript notation $a_{\mathrm{i}}$ used above, we should now denote the variance of $Y_{\mathrm{i}}$ by $a_{\mathrm{ii}} \sigma^{2}$. In this situation, the $Y_{i}$ s may be correlated and have unequal variances as long as the covariances of $Y_{i}$ and $Y_{\mathrm{j}}$ are known except for an unknown scale factor $\sigma^{2}$. In other words, the $a_{\mathrm{ij}} s$ are known but not the factor $\sigma^{2}$. It turns out that the BLUEs in this case are obtained by minimizing a weighted sum of squares which is discussed in Appendix B. Also, for any pair of constants $r_{1}, r_{2}$, the linear combination of estimators $\Sigma r_{\mathrm{j}} \hat{\beta}_{\mathrm{j}}$ is the BLUE of the corresponding linear combination of parameters $\Sigma r_{\mathrm{j}} \beta_{\mathrm{j}}$. It is interesting to note that the unweighted (ordinary) LS estimators are unbiased in this case, but they are not the BLUE's because they are not weighted properly.

\section{INFERENCES}

Under the assumption of independent normal errors with zero mean and constant variance, the probability distributions of the estimators can be derived. The following list provides a summary of the properties:

\section{Distributional Properties}

1. The pair of LS estimators $\left(\hat{\beta}_{0}, \hat{\beta}_{1}\right)$ has a bivariate normal distribution with means, variances and covariance previously stated.

2. The pair $\left(\hat{\beta}_{0}, \hat{\beta}_{1}\right)$ is statistically independent of $s^{2}$.

3. The random variable $(n-2) s^{2} / \sigma^{2}$ has the chi-square distribution with $n-2$ degrees of freedom.

\section{Confidence Limits and Tests}

The distributional properties mentioned above are the basis for deriving confidence intervals and tests of hypotheses about the parameters of the model.

\section{Confidence Limits}

In the following discussion, we denote the $p$ th quantile of the $t$-distribution with $\nu$ degrees of freedom by $t_{p, r}$. That is, $p$ is the area in lower tail of the t-distribution to the left of the value $t_{p, y}$. The following $(1-\alpha) \times 100$ percent confidence limits apply for $\beta_{0}$ and $\beta_{1}$, respectively. 
$\hat{\beta}_{0} \pm t_{1-\alpha / 2, \mathrm{n}-2} S\left\{\Sigma x_{\mathrm{i}}^{2} /\left[n \Sigma\left(x_{\mathrm{i}}-\bar{x}\right)^{2}\right]\right\}^{1 / 2}$,

$\hat{\beta}_{1} \pm t_{1-\alpha /, \mathrm{n}-2} s /\left[\Sigma\left(x_{\mathrm{i}}-\bar{x}\right)^{2}\right]^{1 / 2}$

The $\chi^{2}$-variable in Property 3 , can be used to derive confidence limits for $\sigma^{2}$. In particular, the following are limits for a $(1-\alpha) \times 100$ percent confidence interval for $\sigma^{2}$ :

$\mathrm{LCL}=(n-2) s^{2} / \chi_{1-\alpha / 2, \mathrm{n}-2}^{2}$

$\mathrm{UCL}=(n-2) s^{2} / \chi_{\alpha / 2, \mathrm{n}-2}^{2}$,

where $\chi_{p, y}^{2}$ is the $p$ th quantile of a chi-square distribution with $\nu$ degrees of freedom.

\section{Tests of Hypotheses}

Consider, a test of the null hypothesis $\mathrm{H}_{0}: \beta_{0}=b_{0}$ versus the alternative hypothesis $\mathrm{H}_{2}: \beta_{0} \neq b_{0}$ where $b_{0}$ is some specified number. An appropriate test statistic is $t_{0}=\left[n \sum\left(x_{i}-\bar{x}\right)^{2} / \Sigma x^{2}\right]^{1 / 2}\left(\hat{\beta}_{0}-b_{0}\right) / s$. The test rejects $H_{0}$ in favor of $H_{a}$ if $\left|t_{0}\right| \geqslant t_{1-\alpha / 2, a-2}$. This is known as a two-tailed test, and such a test is appropriate for testing whether a particular $b_{0}$ is a plausible value for $\beta_{0}$ versus all other values either larger or smaller than $b_{0}$. This would be the case when testing whether an intercept coefficient is necessary. For example, if the response variable is measured on a scale with a natural zero point such as length or weight, then it would not make sense to use a model which allows a nonzero intercept. On the other hand, with other types of variables such as temperature measured on either the Celsius or Fahrenheit scales, the intercept depends on which scale is used.

In general, unless there is a compelling reason to have a known intercept (such as zero) in the model, it is best to keep it an arbitrary unknown value denoted by $\beta_{0}$ which is estimated from the data. The test described above with $b_{0}=0$ can be used to decide whether there is statistically significant evidence that the fitted model requires a nonzero intercept.

The SAS output for log-failure rates have a t-value of $t_{0}=-3.917$ with $\nu=107$ degrees of freedom, and a p-value of 0.0002 . This provides strong evidence that a nonzero intercept is needed in the model.

Similarly, a test of the null hypothesis $\mathrm{H}_{0}: \beta_{1}=b_{1}$ versus the alternative hypothesis $\mathrm{H}_{2}: \beta_{1} \neq b_{1}$ where $b_{1}$ is some specified number, tests whether the value $b_{1}$ is a reasonable choice for the slope 
coefficient $\beta_{1}$. An appropriate statistic is $t_{1}=\left[\Sigma\left(x_{i}-\bar{x}\right)^{2}\right]^{1 / 2}\left(\hat{\beta}_{1}-b_{1}\right) / s$. The test rejects $\mathrm{H}_{0}$ in favor of $\mathrm{H}_{\mathrm{a}}$ if $\left|t_{1}\right| \geq t_{1-\alpha / 2, \mathrm{n}-2}$. Again, a two-tailed test is appropriate for testing whether a particular $b_{1}$ is a plausible value for $\beta_{1}$ versus all other values either larger or smaller than $b_{1}$. An important special case of this would involve testing whether the value of a response depends on the covariate. This is when $b_{1}$ $=0$. Formally, the null hypothesis would be $\mathrm{H}_{0}: \beta_{1}=0$. For example, if the response variable is not affected by changes in the covariate, it would not make sense to use a model with a nonzero slope coefficient $\beta_{1}$.

The SAS output for $\log$-failure rates have a t-value of $t_{1}=-4.237$ with $\nu=107$ degrees of freedom, and a p-value of 0.0001 . This provides rather strong evidence that the EDG failure rate is affected by plant age and the model requires a nonzero slope coefficient.

Although one might suspect that the older plants would tend to have the higher failure rates, the data in Example 1 suggests just the opposite. In other words, the higher failure rates tend to be associated with newer plants. This is a good illustration why a two-tailed might be preferred to a onetailed test until the nature of the dependence is well understood.

\section{Testing for a Trend}

There are situations in which it is important to test the value of $\beta_{1}$ against alternatives in a particular direction. For example, consider a test of the null hypothesis $\mathrm{H}_{0}: \beta_{1} \geq b_{1}$ versus the alternative hypothesis $\mathrm{H}_{\mathrm{a}}: \beta_{1}<b_{1}$ where $b_{1}$ is some specified number. This test, which is known as a lower one-sided or lower-tailed test, is a test of whether the value $b_{1}$ is a reasonable value for $\beta_{1}$. An appropriate statistic is the $t$ statistic denoted above by $t_{1}$, but for a lower-tailed test, rejection of $\mathrm{H}_{0}$ in favor of $\mathrm{H}_{\mathrm{a}}$ occurs if $t_{1} \leq-t_{1-\alpha, \mathrm{a}-2}$. Similarly, an upper one-sided or upper-tailed test is a test of the null hypothesis $\mathrm{H}_{0}: \beta_{1} \leq b_{1}$ versus the alternative hypothesis $\mathrm{H}_{2}: \beta_{1}>b_{1}$, and in this case rejection of $\mathrm{H}_{0}$ in favor of $\mathrm{H}_{\mathrm{a}}$ occurs if $t_{1} \geq t_{1-\alpha, \mathrm{n}-2}$.

An important special application deals with the analysis of trends over time in which the direction of the trend (either increasing or decreasing) is specified in the hypothesis. Specifically, the alternative would be of the form $\mathrm{H}_{2}: \beta_{1}>0$ when testing for an increasing trend, and $\mathrm{H}_{2}: \beta_{1}<0$ when testing for a decreasing trend. Strictly speaking, the direction of the alternative hypothesis should be specified prior to observing the data. For example, consider Example 2, which deals with annual radiation exposure in plants for the years 1987-95. Suppose it is suspected, due to increased emphasis on safety training in the nuclear industry that the normalized rate of radiation exposure is decreasing with time. The data are given in Table 1 , and a plot of the data from Table 1 is given in Figure 7. 
Table 1. Radiation exposure (rem), plant years and rate (rem/year), 1987-95.

\begin{tabular}{lllllllllll}
\hline Year & 1987 & 1988 & 1989 & 1990 & 1991 & 1992 & 1993 & 1994 & 1995 \\
\cline { 2 - 9 } Exposure & 39371 & 39610 & 35584 & 36327 & 28352 & 29445 & 26248 & 22088 & 21776 \\
Plant years & 96.00 & 102.00 & 107.25 & 108.00 & 111.00 & 110.25 & 108.00 & 109.00 & 109.00 \\
\cline { 2 - 9 } & 410.12 & 388.33 & 331.79 & 336.36 & 255.42 & 267.08 & 243.04 & 202.64 & 199.78 \\
\hline
\end{tabular}

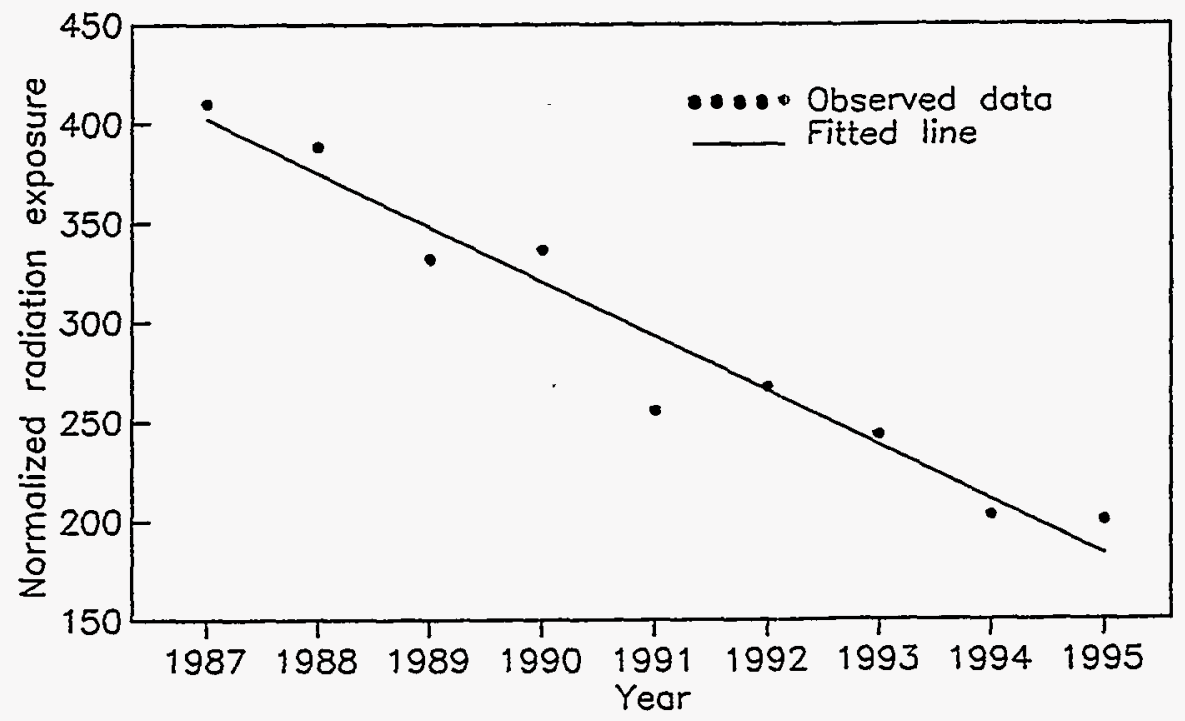

Figure 7. Plot of normalized radiation exposure versus year.

As noted previously, an appropriate alternative when testing against a decreasing trend would be $\mathrm{H}_{\mathrm{a}}: \beta_{1}<0$ (i.e. a negative slope). For this data, a size $\alpha=0.05$ lower one-tailed test of $\mathrm{H}_{0}: \beta_{1} \geq 0$ versus $H_{2}: \beta_{1}<0$ rejects $H_{0}$ if $t_{1} \leq-t_{0.05,7}=-1.895$. The years 1987-1995 have been replaced with coded values 1-9. Such coding, which helps avoid computational problem when estimating intercept, does not effect the estimate of slope. We will make the assumptions of Model (1). Using the SAS 
procedure PROC REG with the data from Table 1, the coefficient of determination is $r^{2}=0.947$, the estimated slope is $\hat{\beta}_{1}=-27.420$, and the t-statistic is $t=-11.212$ which leads to a rejection, indicating a statistically significant decreasing trend. Actually, this corresponds to a $p$-value for a one-tailed test of 0.00005 , which is highly significant. The SAS program and output for these data are given in Appendix A.

It turns out that a slightly better fit is obtained by considering, instead, the logarithmic transformation of the normalized rate of exposure. Using this alternative approach, the coefficient of determination is $r^{2}=0.956$, and the estimate of slope is $\hat{\beta}_{1}=-0.0947$. The corresponding t-statistic is $t=-12.342$.

\section{Confidence Limits for the Mean Response}

One of the most common analyses with the simple linear regression model is to develop a confidence interval for the regression function $\mathrm{E}(Y)=\beta_{0}+\beta_{1} x$. Because the estimate $\hat{y}=\hat{\beta}_{0}+\hat{\beta}_{1} x$ is a linear function of the response data $y_{i}$, it is possible to derive a t-statistic which can be used to construct confidence limits for the regression line. In particular, we have the following $(1-\alpha) \times 100$ percent confidence limits for the expected value of $Y$ at $x, E(Y)=\beta_{0}+\beta_{1} x$ :

$\hat{y} \pm t_{1-\alpha 2, \mathrm{n}-2} s\left[1 / n+(x-\bar{x})^{2} / \Sigma\left(x_{\mathrm{i}}-\bar{x}\right)^{2}\right]^{1 / 2}$.

\section{Simultaneous Confidence Bands}

A very basic, but often misunderstood, aspect of this method is that this interval is derived for a single value of the covariate $x$, and not for more than one covariate value. Thus, if we calculate confidence intervals for two or more values of the covariate, each at a specified confidence level, they will not all be true simultaneously at that specified level. Instead, the confidence intervals only hold point-wise for each value of the covariate $x$. For example, suppose we use the data from Example 1 to construct two $90 \%$ confidence intervals, one with $x=1$ and the other with $x=2$. A conservative confidence coefficient which takes into account the dependence between the intervals can be obtained using the Bonferroni inequality (see Bain and Engelhardt, 1992, p. 16). In particular, this is $1-2(1$ $-0.90)=0.80$. By this approach, we could obtain a conservative confidence coefficient which applies for several of values of $x$. For example, the result for 9 different covariate values is $1-9(1-0.9)=$ 0.10. Clearly, something better is required if we wish to make a reasonable confidence statement which will hold simultaneously for many values of $x$, such as the entire range of plant ages in Example 1 . 
Next we consider a method due to Working, Hotelling and Scheffé for constructing confidence intervals which hold simultaneously for all covariate values and at a prescribed confidence level. This method is discussed by Neter and Wasserman (1974, p.149). Fortunately, the formula for the simultaneous confidence limits differs from those stated above only in replacing the t-quantiles with values which are related to F-quantiles. Specifically, we have

$\hat{y} \pm\left[2 F_{1-\alpha, 2, n-2}\right]^{1 / 2} s\left[1 / n+(x-\bar{x})^{2} / \Sigma\left(x_{i}-\bar{x}\right)^{2}\right]^{1 / 2}$

These confidence intervals are slightly wider, but they hold simultaneously at the $(1-\alpha) \times 100$ percent level for all values of $x$. Because they include the whole regression line $\beta_{0}+\beta_{1} x$ at the stated level, it is reasonable to call the region between the corresponding curves confidence bands. Figure 8 shows the LS regression line for the log-transformed failure rate data of Example 1. The two different types of confidence bands, point-wise and simultaneous, are also shown for comparison.

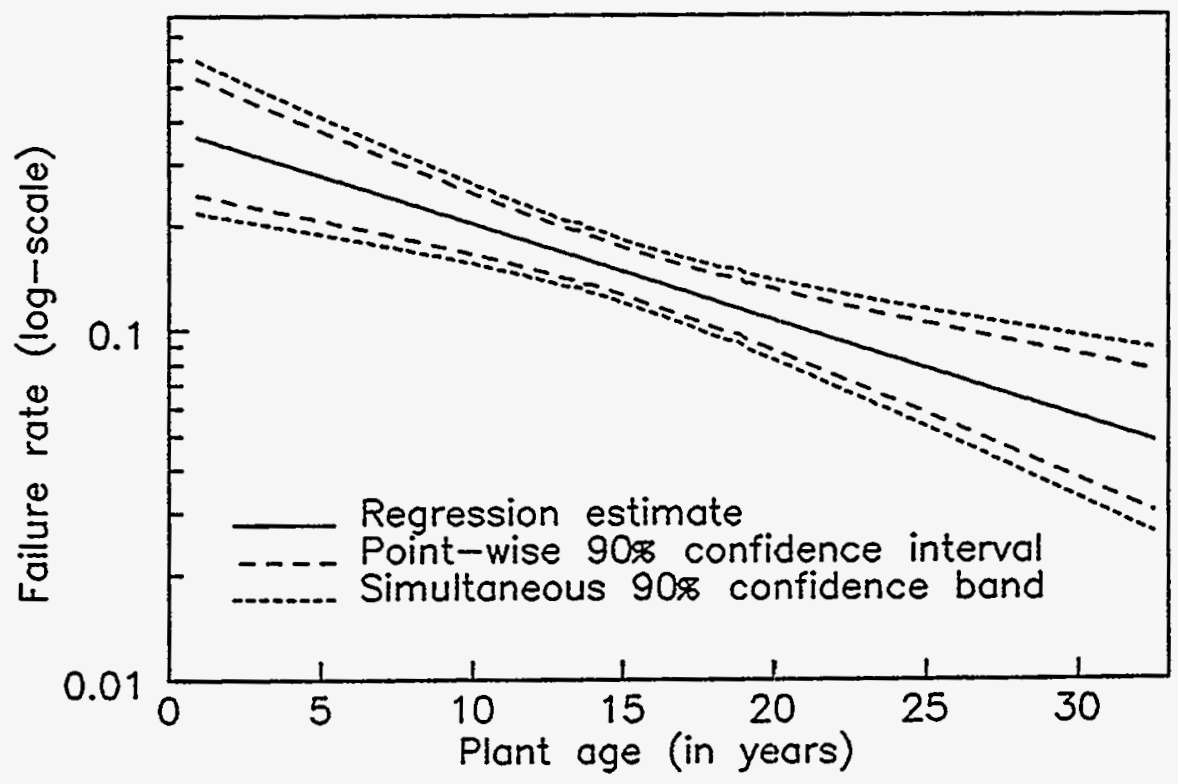

Figure 8. Point-wise limits and simultaneous confidence band for a linear regression.

It should also be noted that another type of confidence band is also sometimes used in a regression analysis. In particular, the estimate $\hat{y}$ for a fixed covariate value $x_{0}$ is sometimes considered as a "predictor" of a future value of the response variable $Y$ with the same value $x_{0}$. In this setting, the 
confidence limits must also take into account the random variation of the future response about its mean value, the regression line itself. Consequently, the resulting intervals, called prediction intervals, are wider than the first confidence intervals for $E(Y)$ described above because they include this additional source of variation. In fact, they are also wider than the simultaneous intervals.

As noted earlier, a logarithmic transformation was required because the diagnostics, applied to the original failure rate data, raised doubts about the assumptions. After carrying out the analysis on the log-data, it will be of interest to transform the results back into the original setting. Figure 9 is a graph of $\exp (\hat{y})$ and the corresponding transformed confidence bands versus $x$.

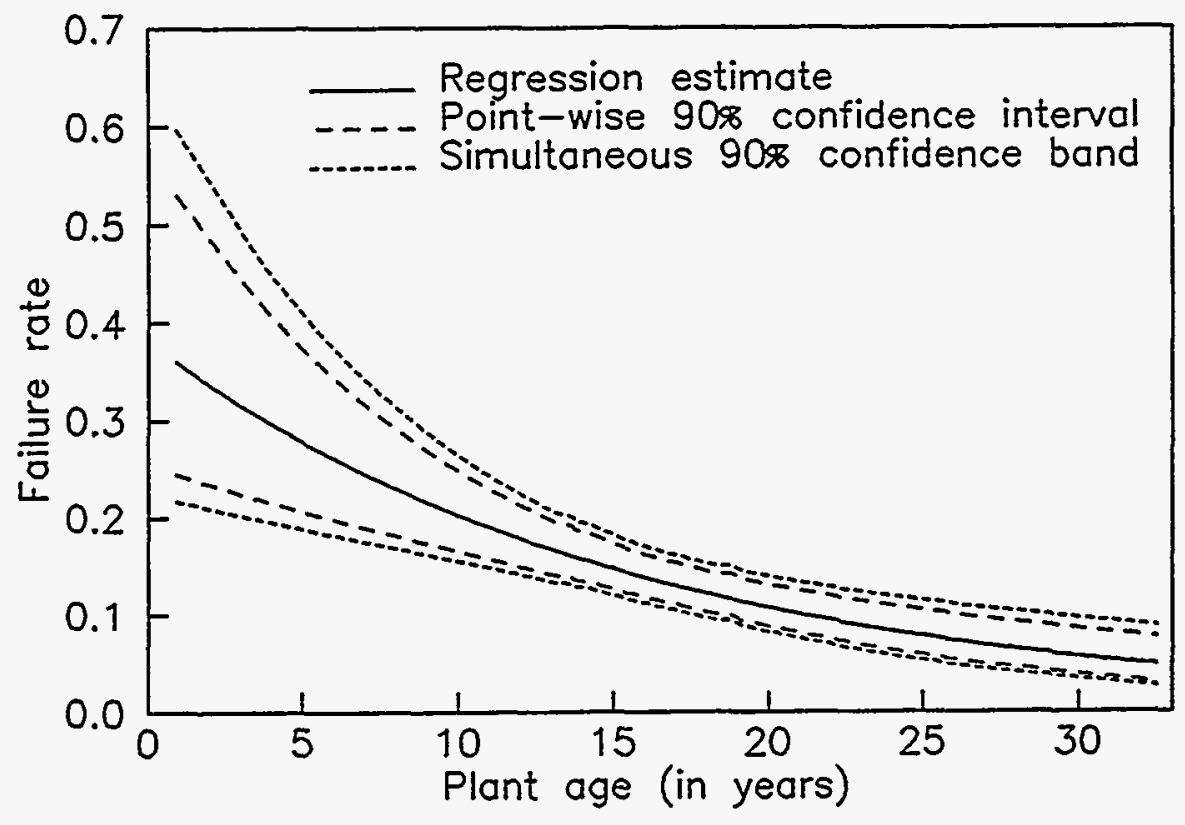

Figure 9. Point-wise limits and simultaneous confidence band for exponential regression.

\section{GENERAL LINEAR MODEL}

Many of the results derived for the simple linear model can be extended to the general linear case in which there is more than one covariate. Consider a linear model of the form

$Y=\beta_{0}+\beta_{1} x_{1}+\cdots+\beta_{\mathrm{m}} x_{\mathrm{m}}+e$ 
where the $\beta_{\mathrm{i}} \mathrm{s}$ are parameters, the $x_{\mathrm{i}} \mathrm{s}$ are covariates and $e$ is a random error with mean of zero. In some applications, the leading term, $\beta_{0}$, is taken to be zero. There is an option in SAS PROC REG which allows the analyst to specify this. However, it is frequently appropriate to have a constant term or $y$ intercept. Another common approach is to have a leading term of $\beta_{0} x_{0}$, where $x_{0}$ is an extra covariate, but to let $x_{0}=1$ when a nonzero $y$-intercept is needed.

Model (4) includes, as a special case, another important example, the polynomial regression model. In this case, the $x_{i} \mathrm{~s}$ are integer powers of the single covariate $x$. In particular, for some $m=$ $1,2, \ldots$

$Y=\beta_{0}+\beta_{1} x+\beta_{2} x^{2}+\cdots+\beta_{\mathrm{m}} x^{\mathrm{m}}+e$.

In the general linear model, multiple observations of the response variable necessitate the use of double subscripts. In particular, if there are $n$ observations of the response variable, and the $i$ th observation is obtained with the covariate values $x_{i 0}, x_{i 1}, \ldots, x_{\mathrm{im}}$, then the notation is

$Y_{\mathrm{i}}=\beta_{0}+\beta_{1} x_{\mathrm{il}}+\cdots+\beta_{\mathrm{mr}} x_{\mathrm{im}}+e_{\mathrm{i}}$.

We will consider the estimation problem in this more general setting.

The LS estimates are the values $\beta_{\mathrm{j}}=\hat{\beta}_{\mathrm{j}}$ that minimize the quantity

$S=\Sigma_{\mathrm{i}}\left[y_{\mathrm{i}}-\Sigma_{\mathrm{j}} \beta_{\mathrm{j}} x_{\mathrm{ij}}\right]^{2}$

The approach used with the simple linear model generalizes readily. In other words, if we equate to zero the partial derivatives of $S$ with respect to the $\beta_{j}$ 's and solve the resulting system of equations we obtain the LS estimates. Specifically, for each of $k=0,1,2, \ldots m$, we equate to zero the partial derivatives

$\partial S / \partial \beta_{k}=\Sigma_{\mathrm{i}} 2\left[y_{\mathrm{i}}-\Sigma_{\mathrm{j}} \beta_{\mathrm{j}} x_{\mathrm{ij}}\right]\left(-x_{\mathrm{ix}}\right)=0$

and solve for the LS estimates. It is not convenient to develop the general model formulas without introducing matrix notation. A discussion of LS estimation with matrix notation is provided for the more ambitious reader in Appendix B. For the remainder of this section, we will discuss the problem in terms of LS estimates which can be obtained using outputs from SAS or some other statistical package such as JMP or Stata.

The estimators $\hat{\beta}_{\mathrm{j}}$ are linear functions of the responses $Y_{\mathrm{i}}$, and it can be shown that they are 
unbiased. As in the case of the simple linear model, the LS estimators of the $\beta_{j} s$ for the general linear model are referred to as the best linear unbiased estimators or BLUEs.

It is also possible to define an unbiased estimator of $\sigma^{2}$, namely

$s^{2}=\Sigma_{i}\left[y_{i}-\Sigma_{j} \hat{\beta}_{j} x_{i j}\right]^{2} /(n-m-1)$

The method of maximum likelihood can be applied if we assume, in addition, that the errors are normal, $\mathrm{N}\left(0, \sigma^{2}\right)$. It is then possible to derive MLEs of the $\beta_{\mathrm{j}} \mathrm{s}$ in a manner similar to what was done in the simple linear case. The ML estimates of the $\beta_{j}$ s are the same as the LS estimates, but the estimate of $\sigma^{2}$ is different, in that it is divided by $n$ rather than $n-m-1$, which makes it biased.

As in the case of the simple linear model, there is a quantity called the error sum of squares,

$\operatorname{SSE}=\Sigma_{\mathrm{i}}\left[y_{\mathrm{i}}-\Sigma_{\mathrm{j}} \hat{\beta}_{\mathrm{j}} x_{\mathrm{ij}}\right]^{2}$

and it is also possible with the general linear model to partition the variation in the same way. If, as before, SST $=\Sigma\left(y_{i}-\bar{y}\right)^{2}$ is the total sum of squares, and SSR $=\Sigma\left(y_{i}-\hat{y}_{i}\right)^{2}$ is the regression sum of squares, then it can be shown that SST = SSR + SSE. Essentially the same rationale as given for the simple linear model can be used to argue that the SSR represents the variation explained by the linear model, and the SSE represents the unexplained variation. Similarly, there is a quantity called the coefficient of determination, $r^{2}=$ SSR/SST, and it can be used as the basis of a formal test of the model. Under the model assumptions previously discussed,

$F=\left[r^{2} / m\right] /\left[\left(1-r^{2}\right) /(n-m-1)\right]$

is F-distributed with $\nu_{1}=m$ and $\nu_{2}=n-m-1$ degrees of freedom. A test of the general linear model has null hypothesis $\mathrm{H}_{0}: \beta_{1}=\beta_{2}=\cdots=\beta_{\mathrm{m}}=0$ versus the alternative hypothesis $\mathrm{H}_{\mathrm{a}}: \beta_{\mathrm{j}} \neq 0$ for at least one $1 \leq j \leq m$, and the test would reject $\mathrm{H}_{0}$ if $F \geq F_{\alpha, m, n-m-1}$. It is also possible, by a modification of this approach, to test parts of the model. This will be discussed later in connection with a related test called the likelihood ratio test.

\section{Confidence Limits and Tests}

Most of the properties of the LS estimates for the simple linear model can be extended; however, the details are more complicated for the general linear case. 


\section{Properties of the Estimators}

Some of the properties of the LS estimates are as follows:

1. The estimators $\hat{\beta}_{0}, \hat{\beta}_{1}, \ldots, \hat{\beta}_{\mathrm{m}}$ have a multivariate normal distribution with means $\beta_{0}, \beta_{1}, \ldots$, $\beta_{\mathrm{m}}$, and covariances $\operatorname{cov}\left(\hat{\beta}_{\mathrm{i}}, \hat{\beta}_{\mathrm{j}}\right)=\sigma^{2} c_{\mathrm{ij}}$ which are given in Appendix $\mathrm{B}$.

2. (n-m-1) $s^{2} / \sigma^{2}$ has a chi-square distribution with $n-m-1$ degrees of freedom.

3. The vector of estimators $\hat{\beta}=\left(\hat{\beta}_{0}, \hat{\beta}_{1}, \ldots, \hat{\beta}_{\mathrm{m}}\right)$ and $s^{2}$ are statistically independent.

4. Each $\hat{\beta}_{j}$ is the BLUE of $\beta_{\mathrm{j}}$.

It is also possible to derive confidence intervals for the parameters. In the following theorem, let $\operatorname{var}\left(\hat{\beta}_{\mathrm{j}}\right)=\sigma^{2} c_{\mathrm{ij}}$. The following are limits for $(1-\alpha) \times 100$ percent confidence intervals for the $\beta_{\mathrm{j}} s$ and $\sigma^{2}:$

$\hat{\beta}_{\mathrm{j}} \pm t_{1-\alpha / 2, n-m-1} s c_{\mathrm{ji}}^{1 / 2}$.

The following are limits for a $(1-\alpha) \times 100$ percent confidence interval for $\sigma^{2}$ :

$L C L=(n-m-1) s^{2} / \chi_{1-\alpha 2 n-m-1}^{2}$

$\mathrm{UCL}=(n-m-1) s^{2} / \chi_{\alpha 2, n-m-1}^{2}$

Consider, for example, a test of the null hypothesis $\mathrm{H}_{0}: \beta_{\mathrm{j}}=b$ versus the alternative hypothesis $\mathrm{H}_{\mathrm{a}}: \beta_{\mathrm{j}} \neq b$ where $b$ is some specified number. Denote by $t_{\mathrm{j}}=\left(\hat{\beta}_{\mathrm{j}}-b\right) /\left(s c_{\mathrm{jj}}^{1 / 2}\right)$. The test rejects $\mathrm{H}_{0}$ in favor of $\mathrm{H}_{2}$ if $\left|t_{\mathrm{j}}\right| \geq t_{1-\alpha / 2, \mathrm{n}-\mathrm{m}-1}$. A two-tailed test is appropriate for testing whether a particular $b$ is a plausible value for $\beta_{\mathrm{j}}$ versus all other values either larger or smaller than $b$. This would be the case when testing whether the responses are actually influenced by the $j$ th covariate (i.e. whether $\beta_{\mathrm{j}}$ differs significantly from zero). Formally, this null hypothesis would be $\mathrm{H}_{0}: \beta_{\mathrm{j}}=0$. Recall, the F-test based on $r^{2}$ can be used to test simultaneously whether all $\beta_{j} s$ differ significantly from zero, whereas the test discussed here tests a single parameter. Similar tests can be defined for testing against either upper or lower one-tailed alternatives.

As an example of a problem involving more than one covariate, we again consider the variables 
of Example 2, with the data given in Table 1. Recall that with our previous approach we were able to convert the problem into a simple linear regression problem by normalizing the radiation exposure data and application of a logarithmic transformation. Thus, the response variable for a given year was $Y=$ $\log$ (exposure/(exposure time)), and the single covariate was coded years 1-9, corresponding to the calendar years 1987-1995.

Suppose we now consider a different approach involving both coded years and plant operating years as separate covariates. The primary purpose for considering this different approach is to illustrate the use of a model with more than one covariate. The model considered now is of the form

$Y_{\mathrm{i}}=\beta_{0}+\beta_{1} x_{\mathrm{i} 1}+\beta_{2} x_{\mathrm{i} 2}+e_{\mathrm{i}}$

where, for the $i$ th calendar year, the $Y_{\mathrm{i}}$ is the log transformation of the radiation exposure, $x_{\mathrm{i} 1}=i$ and $x_{i 2}$ is the logarithm of exposure time. The SAS procedure PROC REG could be used to compute the estimates needed in this analysis, but to illustrate the use of other, more interactive statistical software packages, we have done the computations using the program STATA 4.0. A log of the output is also included in Appendix A. The estimates, based on the data of Table 1, are $\hat{\beta}_{0}=7.665, \hat{\beta}_{1}=-0.091$ and $\hat{\beta}_{2}=0.665$. The coefficient of determination for this model is $r^{2}=0.945$, and the corresponding F-statistic given by Equation (8) is 51.62. The degrees of freedom for this test are 2 for the numerator and 6 for the denominator, leading to a p-value of 0.0002 . The t-statistic associated with the slope is $t$ $=-7.567$ with $\nu=6$ degrees of freedom, corresponding to a p-value of 0.0001 .

The choice between modeling the log-normalized radiation with one covariate (Model 1) versus modeling log-radiation with two covariates (Model 2) is not completely obvious. Although the Model 1 has a slightly larger coefficient of variation, there are some other aspects of the models which favor Model 2. For example, concerning the assumption of normality, the residuals for Model 2 conform more closely with a normal assumption. Normal probability plots such as Figure 6 could be used to verify this, but a simpler way would be to compare p-values for goodness-of-fit tests of normality applied to the residuals from each model. The Shapiro-Wilk tests of normality yield p-values of 0.48 and 0.88 , respectively for Models 1 and 2. The p-value for Model 1 is smaller, but if the customary 0.05 level is used, neither test is close to being significant. Because of its simplicity, Model 1 would be recommended for use in this example.

Another question of interest involves joint tests and confidence statements about two or more of the coefficients. The above discussion about tests for parameters of the general linear model provides a method for testing individual regression coefficients with the other parameters of the model regarded as unknown nuisance parameters. It is also possible to develop methods for simultaneously testing two 
or more of the regression coefficients.

In a more general setting, we consider an observable random variable $Y$ with probability density function $f(y ; \theta)$ where $\theta$ is a parameter of a model. If there are multiple parameters, then subscripts are used and the parameters are represented as components of a vector with a bold font notation, $\theta=\left(\theta_{1}\right.$, $\theta_{2}, \ldots, \theta_{\mathrm{k}}$ ). If there is only a single parameter, then it is written as $\theta$ (without a subscript and not bold). There may be restrictions on some of the parameters. For example, if the ith parameter is a variance, then it is required to be positive, $\theta_{i}>0$. The parameter space for this model is the $k$-dimensional set $\Omega$ consisting of all such vectors $\theta$.

In mathematical statistics, a hypothesis is defined to be a statement about the distribution of $Y$. When the distribution involves a parameter $\theta$, the hypothesis can often be expressed in terms of some subset of the parameter space, say $\Omega_{0} \subset \Omega$. For example, in the simple linear model, the parameter space (including the variance of the error) consists of all triples $\theta=\left(\beta_{0}, \beta_{1}, \sigma^{2}\right)$ such that $-\infty<\beta_{\mathrm{j}}<\infty$ for $j=0$ or 1 and $\sigma^{2}>0$. The null hypothesis $\mathrm{H}_{0}: \beta_{1}=0$ would correspond to the subset $\Omega_{0}$ consisting of all triples of the form $\left(\beta_{0}, 0, \sigma^{2}\right)$. Geometrically, one could associate $\Omega_{0}$ with a plane (a twodimensional subset of the three-dimensional set $\Omega$ ). As another illustration, consider the hypothesis that the mean response at a fixed covariate value has a specified value, say $E(Y \mid x=5)=2$. In other words, $\mathrm{H}_{0}: \beta_{0}+\beta_{1} 5=2$. Again, we can associate the hypothesis with a two-dimensional subset of the parameter space $\Omega$, although it is slightly more complicated than the first example.

A rather general approach to this problem is to construct a test called the Likelihood Ratio Test (LRT). Let $\Omega_{0}$ be a subset of $\Omega$, and consider the null hypothesis that $\theta$ is contained in $\theta_{0}$ versus the alternative that $\theta$ is contained in $\Omega-\Omega_{0}$ (the complement of $\Omega_{0}$ in $\Omega$ ). That is, the null hypothesis is $\mathrm{H}_{0}$ : $\theta \in \Omega_{0}$ versus an alternative of the form $H_{2}: \theta \in \Omega-\Omega_{0}$. The likelihood ratio statistic is

$$
\Lambda=\frac{\max _{\theta \in \Omega_{0}} L(\theta)}{\max _{\theta \in \Omega} L(\theta)} .
$$

where $L(\theta)=\Pi_{j} f\left(\gamma_{i} ; \theta\right)$ is the likelihood function for data from $f(y ; \theta)$. The form of the LRT is to reject $\mathrm{H}_{0}$ if $\Lambda \leq c$ where $c$ is a constant chosen to provide a test of size $\alpha$. The general principle behind the LRT is to reject when the observed data are much less likely under the null hypothesis than they are without this restriction. Notice that the denominator of $\Lambda$ is the likelihood function evaluated at the MLEs over the unrestricted space $\Omega$, whereas the numerator is the likelihood function evaluated at the MLEs over the restricted space $\Omega_{0}$. 
The determination of an appropriate value $c$ can be a major problem in itself, but a convenient approximate test can be used in many cases. In particular, if regularity conditions hold which ensure that the MLEs are asymptotically normally distributed, then the asymptotic distribution of $\Lambda$ is free of unknown nuisance parameters, and under the null hypothesis $\mathrm{H}_{0}$, the asymptotic distribution of $-2 \log (\Lambda)$ is $\chi^{2}$ with degrees of freedom equal to the difference in dimensions of $\Omega$ and $\Omega_{0}$. For example, each null hypothesis discussed above with the simple linear model has degrees of freedom $3-2=1$, and $\mathrm{H}_{0}$ would be rejected at approximate level $\alpha$ if $-2 \log (\Lambda) \geq \chi_{\alpha, 1}^{2}$.

To further illustrate the LRT approach, we consider an extension of the modeling already done based on the data of Example 1. Suppose instead of the linear fit to the log-data, we wish to fit a second degree polynomial. We are not actually advocating a quadratic fit, but are merely using this as a hypothetical example to illustrate likelihood ratio tests. So the proposed model is

$Y=\beta_{0}+\beta_{1} x+\beta_{2} x^{2}+e$

where $e$ is normal with mean 0 and variance $\sigma^{2}$. Including the variance, there are four unknown parameters, which can be represented in vector notation as an ordered quadruple, $\theta=\left(\beta_{0}, \beta_{1}, \beta_{2}, \sigma^{2}\right)$. The parameter space for this model, denoted by $\Omega$, is the set of all such quadruples with $-\infty<\beta_{\mathrm{j}}<$ $\infty$, and $\sigma^{2}>0$. Although the plot of the data in Figure 4 suggests that some sort of regression model is appropriate, it would be desirable, in general, to test whether terms beyond the constant term $\beta_{0}$ are really needed. In other words, a test is sought for whether linear and second degree terms really explain enough of the variation in the data to be useful. Note that there is always a trade-off between the explanatory value of extra terms and the complexity they add to the model. The above results about testing of hypotheses can be used for testing individual parameters, say $\mathrm{H}_{0}: \beta_{1}=0$ or $\mathrm{H}_{0}: \beta_{2}=0$, but it would be desirable to test both parameters simultaneously, $\mathrm{H}_{0}: \beta_{1}=\beta_{2}=0$. Such a test can be constructed using the LRT. The test compares the simultaneous hypothesis about $\beta_{1}$ and $\beta_{2}$ mentioned above versus the alternative that at least one of these coefficients is nonzero. The parameters $\beta_{0}$ and $\sigma^{2}$ are unknown nuisance parameters for this test. Let $\Omega$ be the parameter space discussed above, and let $\Omega_{0}$ be the subset of $\Omega$ consisting of points $\left(\beta_{0}, 0,0, \sigma^{2}\right)$. Over the subspace $\Omega_{0}$, the MLEs of $\beta_{0}$ and $\sigma^{2}$ are, respectively $\bar{y}$ and $\hat{\sigma}_{0}^{2}=\Sigma\left(y_{\mathrm{i}}-\bar{y}\right)^{2} / n$, while over the unrestricted space $\Omega$ the joint MLEs are the estimates (6) and

$\hat{\sigma}^{2}=\Sigma\left(y_{i}-\hat{y}_{i}\right)^{2} / n$

with $m=2, x_{i 1}=x_{i}$ and $x_{i 2}=x_{i}^{2}$.

After some algebra, the likelihood ratio can be expressed in terms of the MLEs as 
$\Lambda=\left(\hat{\sigma}_{0}^{2} / \hat{\sigma}^{2}\right)^{-n / 2}$.

For example, the LRT would reject at level $\alpha=0.05$ if $-2 \log (\Lambda)=n \log \left(\hat{\sigma}_{0}^{2} / \hat{\sigma}^{2}\right) \geq \chi_{0.05,2}^{2}=5.99$. The degrees of freedom are $4-2=2$, because the parameter space is 4-dimensional, and under $\mathrm{H}_{0}$ the dimension is 2 .

In this case, a related test can be derived. In particular, notice that $\hat{\sigma}_{0}^{2} / \hat{\sigma}^{2}=($ SST $/ n) /(\mathrm{SSE} / n)=$ SST/SSE, where SST and SSE are the total sums of squares and the error sum of squares discussed previously in the report. This, in turn, is related to the test which was discussed earlier in connection with the coefficient of determination, $r^{2}=$ SSR/SST $=1-$ SSE/SST. Thus, a large value of $r^{2}$ corresponds to a small value of $\Lambda$, and the LRT and the F-test of (8) are related. The F-test has the advantage of having a known distribution, whereas application of the LRT relies on an large-sample $\chi^{2}$ approximation.

\section{Confidence Contours}

It is also possible to make joint confidence statements about the $\beta_{j}$ s. Consider the sum $S=S(\beta)$ $=\Sigma_{\mathrm{i}}\left[y_{\mathrm{i}}-\Sigma_{j} \beta_{j} x_{i j}\right]^{2}$, defined by Equation (5) with response values $y_{1}, y_{2}, \ldots, y_{\mathrm{n}}$ and $\beta=\left(\beta_{0}, \beta_{1}, \ldots\right.$, $\beta_{m}$ ). This is the sum of squares that was minimized to obtain the LS estimates of the regression coefficients. In other words, the minimum value of $S$ is $S(\hat{\beta})$. If $Y_{1}, Y_{2}, \ldots, Y_{\mathrm{a}}$ are independent random variables which satisfy the conditions of Model (4) (i.e. the general linear model), it can be shown that $s^{2}$ and $S(\beta)-S(\hat{\beta})=\Sigma_{i}\left[Y_{i}-\Sigma_{j} \beta_{j} x_{j i}\right]^{2}-\Sigma_{i}\left[Y_{i}-\Sigma_{j} \hat{\beta}_{j} x_{i j}\right]^{2}$ are independent random variables, and furthermore, that $[S(\beta)-S(\hat{\beta})] / \sigma^{2}$ is $\chi^{2}$ with $m+1$ degrees of freedom. As noted previously, $(n-m$ $-1) s^{2} / \sigma^{2}$ has a $\chi^{2}$ distribution with $n-m-1$ degrees of freedom. Thus, the variable

$F=[S(\beta)-S(\hat{\beta})] /[(m+1) s]$

has an $\mathrm{F}$ distribution with $m+1$ and $n-m-1$ degrees of freedom. Thus, the following inequality holds with probability $1-\alpha$,

$S(\beta)-S(\hat{\beta}) \leq(m+1) s F_{1-\alpha, m+1, a-m-1}$

This inequality defines an $(m+1)$-dimensional region called a $(1-\alpha) \times 100$ percent confidence region for the regression coefficients $\beta=\left(\beta_{0}, \beta_{1}, \ldots, \beta_{\mathrm{m}}\right)$. The boundary of such a region is known as a confidence contour. For the important special case of the simple linear model, the corresponding 
confidence contour is an ordinary two-dimensional ellipse with center at $\left(\beta_{0}, \hat{\beta}_{1}\right)$. For the purpose of illustration, a 95 percent confidence contour based on the data of Table 1 is shown in Figure 10. The years have been coded so that 1987 corresponds to $x=1,1988$ to $x=2$, and so on. This topic is

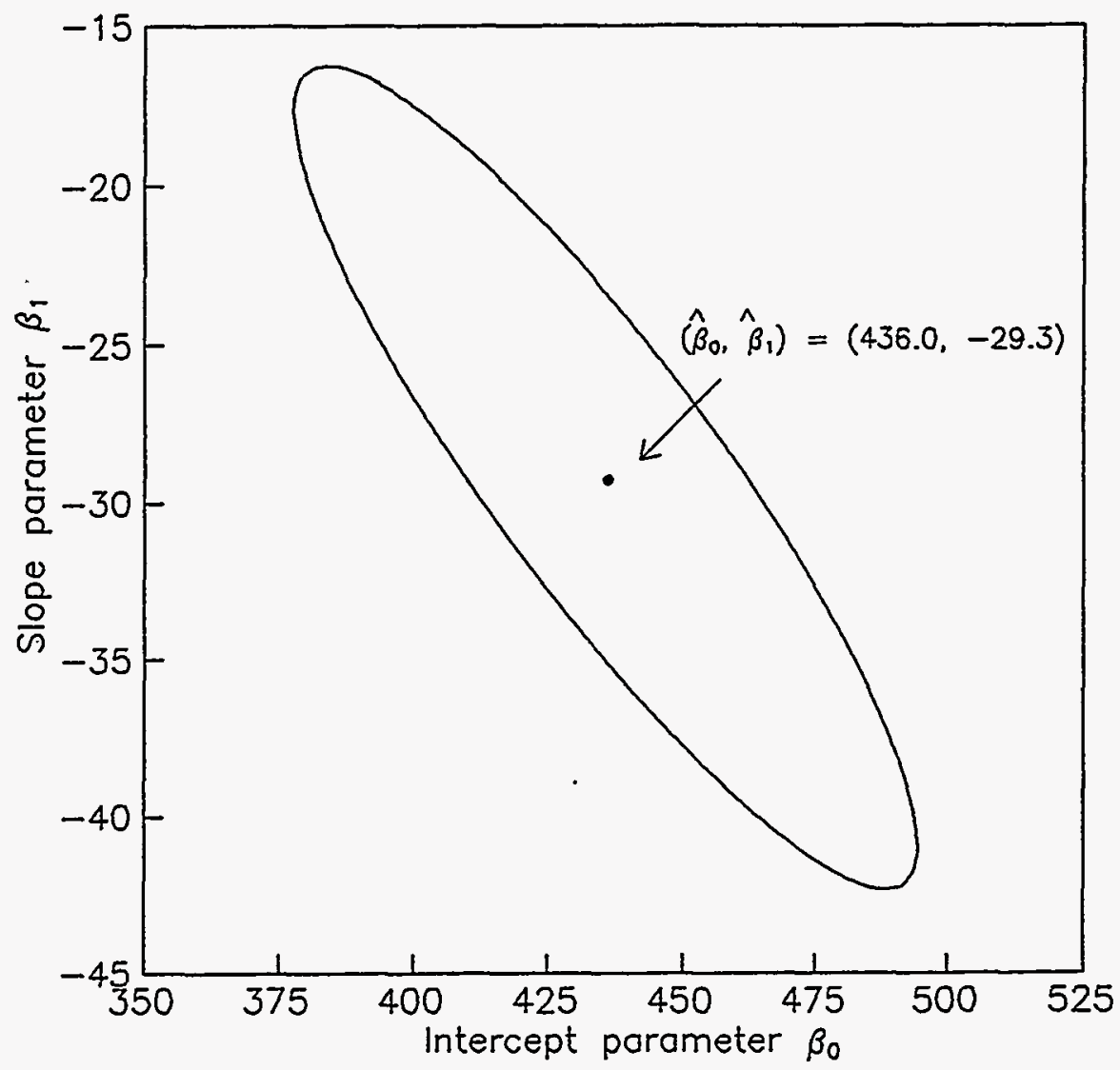

Figure 10. $95 \%$ confidence contour for regression coefficients.

discussed in more detail in Appendix B.

\section{Testing Parts of a Model}

It is also desirable to be able to test whether one or more covariates of a general linear model can 
be eliminated. This will be accomplished by means of a joint test of whether all coefficients in some subset differ significantly from zero. Assume the general linear model (i.e. Model (4)) with $m$ covariates, $x_{1}, x_{2}, \ldots, x_{\mathrm{m}}$ and $m+1$ coefficients $\beta_{0}, \beta_{1}, \ldots, \beta_{\mathrm{m}}$. Consider a null hypothesis of the form $\mathrm{H}_{0}: \beta_{\mathrm{p}}=$ $\cdots=\beta_{\mathrm{m}}=0$ for some $0 \leq p \leq m$, against the alternative that at least one of these is non-zero. The first $p$ coefficients $\beta_{0}, \ldots, \beta_{\mathrm{p}-1}$ and $\sigma^{2}$ are unknown and unspecified for this test. Denote by $\Omega$ the set of all $(m+2)$-tuples $\left(\beta_{0}, \beta_{1}, \ldots, \beta_{\mathrm{m}}, \sigma^{2}\right)$ with $-\infty<\beta_{\mathrm{j}}<\infty$, and let $\Omega_{0}$ be the subset of $\Omega$ such that $\beta_{\mathrm{j}}=0$ for $p \leq j \leq m$. The MLEs over the unrestricted space $\Omega$ are the estimates defined by Equation (6) and by $\hat{\sigma}^{2}=S(\hat{\beta}) / n$. On the other hand, the MLEs over the restricted space $\Omega_{0}$ are the same as the MLEs for a lower-dimensional model. Consider now the (unrestricted) MLE problem for the lowerdimensional general linear model with $p$ coefficients $\beta_{0}, \beta_{1}, \ldots \beta_{\mathrm{p}-1}$. The MLEs would be the solutions to a subset of equations defined by (6); that is with $0 \leq k \leq p-1$, and these solutions would be identical to the MLEs of the $\beta_{j} s$ over the restricted subspace $\Omega_{0}$ of the higher-dimensional problem discussed above. We represent these solutions as components of a $p$-dimensional vector, $\hat{\beta}_{0}$, The MLEs of $\sigma^{2}$ would also be identical, denoted here by $\hat{\sigma}_{0}^{2}=S\left(\hat{\beta}_{0}\right) / n$. With this notation, the LRT has the same form as (9), namely

$\Lambda=\left(\hat{\sigma}_{0}^{2} / \hat{\sigma}^{2}\right)^{-\mathrm{n} / 2}$.

The LRT would reject at the approximate level $\alpha$ if

$$
-2 \log (\Lambda)=n \log \left(\hat{\sigma}_{0}^{2} / \hat{\sigma}^{2}\right) \geq \chi_{\alpha, m-p+1}^{2}
$$

The number of degrees of freedom is the difference in the dimensions of $\Omega$ and $\Omega_{0}$, namely $(m+2)-$ $(p+1)=m-p+1$.

As in the example of the fitted quadratic model, an F-test can be derived. In particular, let SSE $=S\left(\hat{\beta}_{0}\right)$ be the sum of squares associated with the restricted space $\Omega_{0}$ and let $S_{S E}=S(\hat{\beta})$ be the sum of squares for the unrestricted space $\Omega$. Because $S S E_{1}$ is obtained by minimizing the sum $S(\beta)$ over a subset of $\Omega$, and $S_{S E}$ is obtained by minimizing the same function over the whole set, then necessarily $S_{S E}$ $\geq \mathrm{SSE}_{2}$. In other words, by reducing the number of terms in the model, the error sum of squares is increased. One way to interpret this is that there is less variation explained by the reduced model than by the full model. It is also possible to get a quantitative assessment of this reduction with a test based on the difference $S_{S E}-S_{1} E_{2}$. Using advanced theory about such sums of squares, it is possible to show that when $\beta_{\mathrm{j}}=0$ for all $p \leq j \leq m$, the difference $\mathrm{SSE}_{1}-\mathrm{SSE}_{2}$ is independent of $S S E_{2}$, and furthermore each of these quantities has a $\chi^{2}$ distribution. In particular, $S_{S E_{1}}-S_{S E}$ is $\chi^{2}$-distributed with $m-p-1$ degrees of freedom, and $\mathrm{SSE}_{2}$ is $\chi^{2}$-distributed with $n-m-1$. Consequently, an $\mathrm{F}$ statistic can be constructed, namely 
$F=\left[\left(\mathrm{SSE}_{1}-\mathrm{SSE}_{2}\right) /(m-p-1)\right] /\left[\mathrm{SSE}_{2} /(n-m-1)\right]$,

which has the F-distribution with $m-p-1$ and $n-m-1$ degrees of freedom. Because a large difference $\mathrm{SSE}_{1}-\mathrm{SSE}_{2}$ indicates a large amount of variation explained by retaining the extra terms, a large value of $F$ supports the use of the full model. A size $\alpha$ test of $\mathrm{H}_{0}: \beta_{\mathrm{p}}=\cdots=\beta_{\mathrm{m}}$ against the alternative that $\beta_{\mathrm{i}} \neq \beta_{\mathrm{j}}$ for at least one pair $i \neq j$ is to reject $\mathrm{H}_{0}$ if

$F \geq F_{1-\alpha, m-p-1, n-m-1}$

Notice that this is a generalization of the F-test defined by (8) for testing whether parameters beyond the intercept $\beta_{0}$ are significant.

Notice also that $\mathrm{SSE}_{1}=n \hat{\sigma}_{0}^{2}$ and $\mathrm{SSE}_{2}=n \hat{\sigma}$, so that the F-test is related to the LRT. Specifically, the LRT is based on the ratio of the restricted MLE to the unrestricted MLE of $\sigma^{2}$,

$\hat{\sigma}_{0}^{2} / \hat{\sigma}^{2}=\mathrm{SSE}_{1} / \mathrm{SSE}_{2}$

Consequently, a large value of $F$ corresponds to a large value of $-2 \log (\Lambda)=n \log \left(\hat{\sigma}_{0}^{2} / \hat{\sigma}^{2}\right)$. Thus, the F-test defined by $(10)$ is related to the LRT. The LRT uses a $\chi^{2}$ approximation, while (10) is Fdistributed and for this reason, (10) is preferable.

\section{Selecting Covariates}

A great deal has been written about techniques for selecting covariates to use in a regression problem. One possibility would be to start out with a large set of covariates all of which might be useful in explaining variation and then try to choose a best set by looking at all possible subsets. There are also techniques for choosing a "best" subset where best is defined in terms of some numerical measure such as $r^{2}$. Algorithms for looking at all possible regressions and selecting best subsets of covariates are discussed by Christensen (1987, p.283). Such an analysis can be carried out with the SAS procedure PROC RSQUARE.

\section{Stepwise Model Selection}

Another method of selecting which covariates to use is known as stepwise regression. There are two basic procedures used in stepwise regression: forward selection and backward elimination. It is possible to use these options individually or a combination of both. 
Forward selection sequentially adds more variables to a model until a prescribed stopping rule is satisfied. Because this is a sequential procedure, the model changes at each step. For example, one common procedure is to add the variable that increases $r^{2}$ the most. This can be carried out with the SAS procedure PROC STEPWISE. This procedure begins by finding the variable that produces the largest $r^{2}$. In the second step, the procedure finds that variable which, when added to the already selected variable, results in the largest increase in $r^{2}$. In the third step, the procedure finds that variable which, when added to the two already selected results in the largest increase in $r^{2}$, and so on. The process continues until no variable considered for addition to the model provides a statistically significant improvement in the model fit, as measured by the corresponding F-test. For example, suppose at some stage we have built a simple linear model $E(Y)=\beta_{0}+\beta_{1} x_{1}$, and the next step is to choose between two covariates $x_{2}$ and $x_{3}$ with associated parameters $\beta_{2}$ and $\beta_{3}$. The procedure would be to compute F-tests as defined by (10) for both $\mathrm{H}_{0}: \beta_{2}=0$ and $\mathrm{H}_{0}: \beta_{3}=0$, and add the covariate with the smaller of the $p$ values provided the test is significant. At each step, the $p$-value which is used to order the variables considered for inclusion in the next model will depend on the model which resulted from the previous step. Once a variable is added by forward selection, it remains in the model.

Backward elimination begins by computing the regression with all independent variables. The $p$-values for F-tests performed for all individual terms are examined to find the coefficient that contributes the least to the fit of the model, that is, the one with the largest $p$-value. This term is deleted from the model and the backward elimination process is applied to the reduced model, and so on. This procedure stops when all coefficients remain in the model are statistically significant. Once a variable is deleted by backward elimination, it never reenters the model.

The stepwise procedure is a combination of both forward and backward procedures. Stepwise selection begins like forward selection, but after a covariate has been added, the resulting model is examined to see if any coefficient has a sufficiently large $p$-value to justify use of backward elimination. Then the forward selection is implemented again. This procedure continues until no additions or deletions are indicated according to a prescribed significance level. It should be noted that the prescribed significance level applies at each individual step, and any claim of significance for the model which is finally selected would have to be less because many such tests must be made in the process of building the model. As noted, the procedure can be carried out with the SAS procedure PROC STEPWISE. 


\section{REFERENCES}

Atwood, Corwin L. (1994), "Hits per Trial: Basic Analysis of Binomial Data," EGG-RAAM-11041.

Bain, Lee J. and Engelhardt, Max (1992), Introduction to Probability and Mathematical Statistics, 2nd ed., Boston: PWS-Kent.

Christensen, R. (1987), Plane Answers to Complex Questions: The Theory of Linear Models, Berlin: Springer-Verlag.

D'Agostino, R. B. and Stephens, M. A. (1986), Goodness-of-Fit Techniques, New York: Marcel Dekker.

Galton, G., (1889), Natural Inheritance, London: Macmillan.

Gauss, C. F., (1823), Theoria Combinationis Observationum Erroribus Minimis Obnoxiae, Göttingen: Dietrich.

SAS Institute Inc. (1988), SAS/STAT User's Guide, Release 6.03 Edition, Cary, NC.

Annual Report for 1992, Office for Analysis and Evaluation of Operational Data, Power Reactors, NUREG-1272, Vol. 7, No. 1, July 1993. 
Appendix A: Statistical Computation

A-1 


$$
\text { A-2 }
$$




\section{Appendix A}

\section{Statistical Computation}

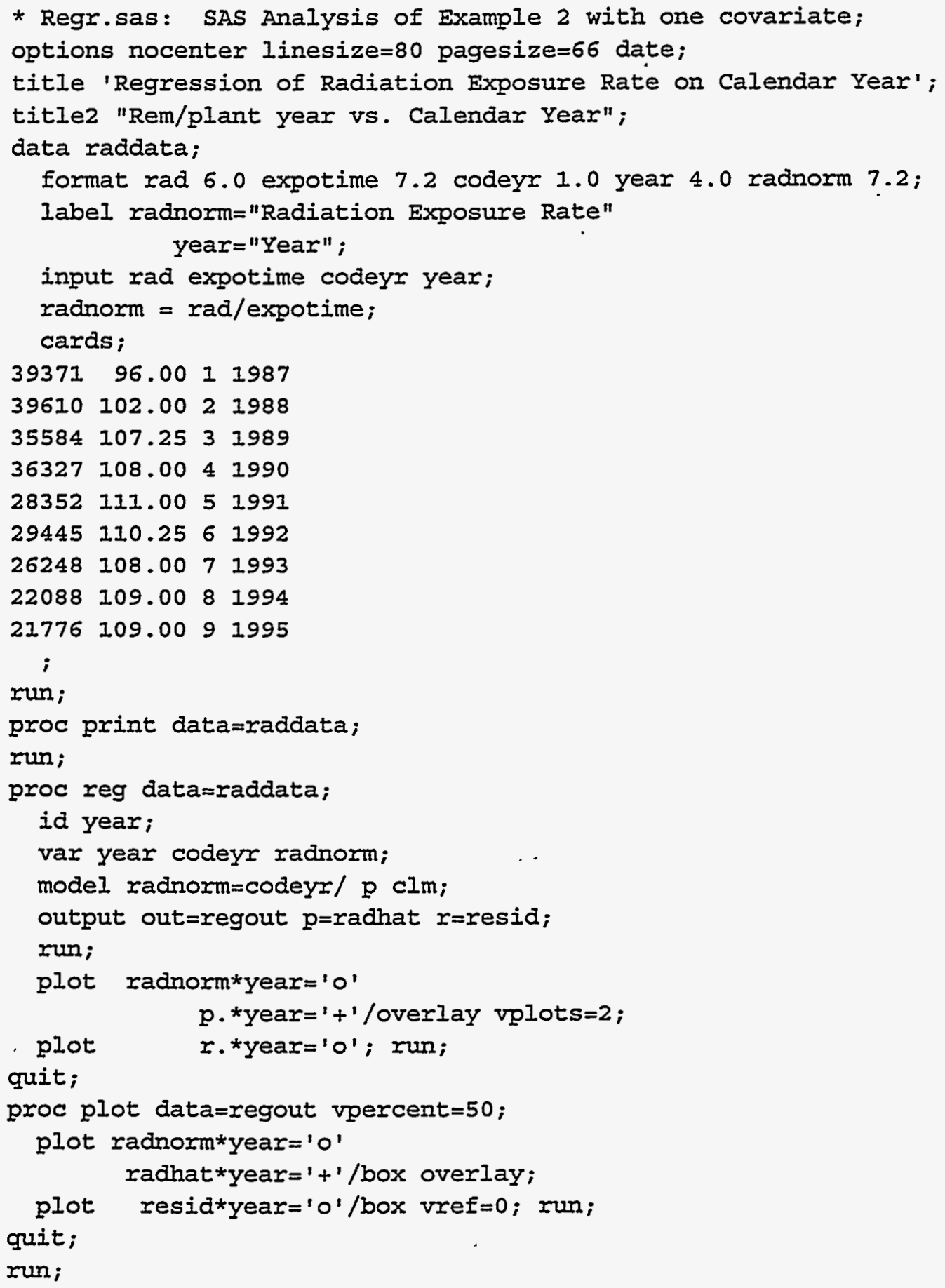




\section{Output}

Regression of Radiation Exposure Rate on Calendar Year Rem/plant year vs. Calendar Year

$\begin{array}{rrrrrr}\text { OBS } & \text { RAD } & \text { EXPOTIME } & \text { CODEYR } & \text { YEAR } & \text { RADNORM } \\ & & & & & \\ 1 & 39371 & 96.00 & 1 & 1987 & 410.11 \\ 2 & 39610 & 102.00 & 2 & 1988 & 388.33 \\ 3 & 35584 & 107.25 & 3 & 1989 & 331.79 \\ 4 & 36327 & 108.00 & 4 & 1990 & 336.36 \\ 5 & 28352 & 111.00 & 5 & 1991 & 255.42 \\ 6 & 29445 & 110.25 & 6 & 1992 & 267.07 \\ 7 & 26248 & 108.00 & 7 & 1993 & 243.04 \\ 8 & 22088 & 109.00 & 8 & 1994 & 202.64 \\ 9 & 21776 & 109.00 & 9 & 1995 & 199.78\end{array}$

MOdel : MODELI

Dependent Variable: RADNORM Radiation Exposure Rate

Analysis of variance

$\begin{array}{lrrrrr}\text { Source } & \text { DF } & \begin{array}{r}\text { Sum of } \\ \text { Squares }\end{array} & \begin{array}{r}\text { Mean } \\ \text { Square }\end{array} & \text { F Value } & \text { Prob>F } \\ \text { Model } & 1 & 45111.15172 & 45111.15172 & 125.713 & 0.0001 \\ \text { Error } & 7 & 2511.90262 & 358.84323 & & \\ \text { C Total } & 8 & 47623.05435 & & & \end{array}$

$\begin{array}{lrlr}\text { Root MSE } & 18.94316 & \text { R-square } & 0.9473 \\ \text { Dep Mean } & 292.72799 & \text { Adj R-sq } & 0.9397 \\ \text { C.V. } & 6.47125 & & \end{array}$

Parameter Estimates

\begin{tabular}{|c|c|c|c|c|c|}
\hline Variable & $\mathrm{DF}$ & $\begin{array}{r}\text { Parameter } \\
\text { Estimate }\end{array}$ & $\begin{array}{r}\text { standard } \\
\text { Error }\end{array}$ & $\begin{array}{c}T \text { for Ho: } \\
\text { Parameter }=0\end{array}$ & Prob $>|\mathrm{T}|$ \\
\hline INTERCEP & 1 & 429.827634 & 13.76188517 & 31.233 & 0.0001 \\
\hline CODEYR & 1 & -27.419929 & 2.44555117 & -11.212 & 0.0001 \\
\hline Variable & $D F$ & $\begin{array}{r}\text { Variable } \\
\text { Label }\end{array}$ & & & \\
\hline INTERCEP & 1 & Intercept & & & \\
\hline CODEYR & 1 & & & & \\
\hline
\end{tabular}




$\begin{array}{rrrrrrrr}\text { Obs } & \text { YEAR } & \begin{array}{r}\text { Dep Var } \\ \text { RADNORM }\end{array} & \begin{array}{r}\text { Predict } \\ \text { Value }\end{array} & \begin{array}{r}\text { Std Err } \\ \text { Predict }\end{array} & \begin{array}{r}\text { Lower95\% } \\ \text { Mean }\end{array} & \begin{array}{r}\text { Upper95\% } \\ \text { Mean }\end{array} & \text { Residual } \\ 1 & & & & & & & \\ 2 & 1987 & 410.1 & 402.4 & 11.643 & 374.9 & 429.9 & 7.7069 \\ 3 & 1988 & 388.3 & 375.0 & 9.680 & 352.1 & 397.9 & 13.3456 \\ 4 & 1989 & 331.8 & 347.6 & 7.987 & 328.7 & 366.5 & -15.7823 \\ 5 & 1991 & 336.4 & 320.1 & 6.771 & 304.1 & 336.2 & 16.2132 \\ 6 & 1992 & 255.4 & 292.7 & 6.314 & 277.8 & 307.7 & -37.3046 \\ 7 & 1993 & 243.0 & 237.9 & 7.987 & 219.0 & 256.8 & 5.1489 \\ 8 & 1994 & 202.6 & 210.5 & 9.680 & 187.6 & 233.4 & -7.8260 \\ 9 & 1995 & 199.8 & 183.0 & 11.643 & 155.5 & 210.6 & 16.7315\end{array}$

Sum of Residuals

Sum of Squared Residuals

Predicted Resid SS (Press)
$-2.55795 E-13$
2511.9026
3833.4547 
Regression of Radiation Exposure Rate on Calendar Year Rem/plant year vs. Calendar Year

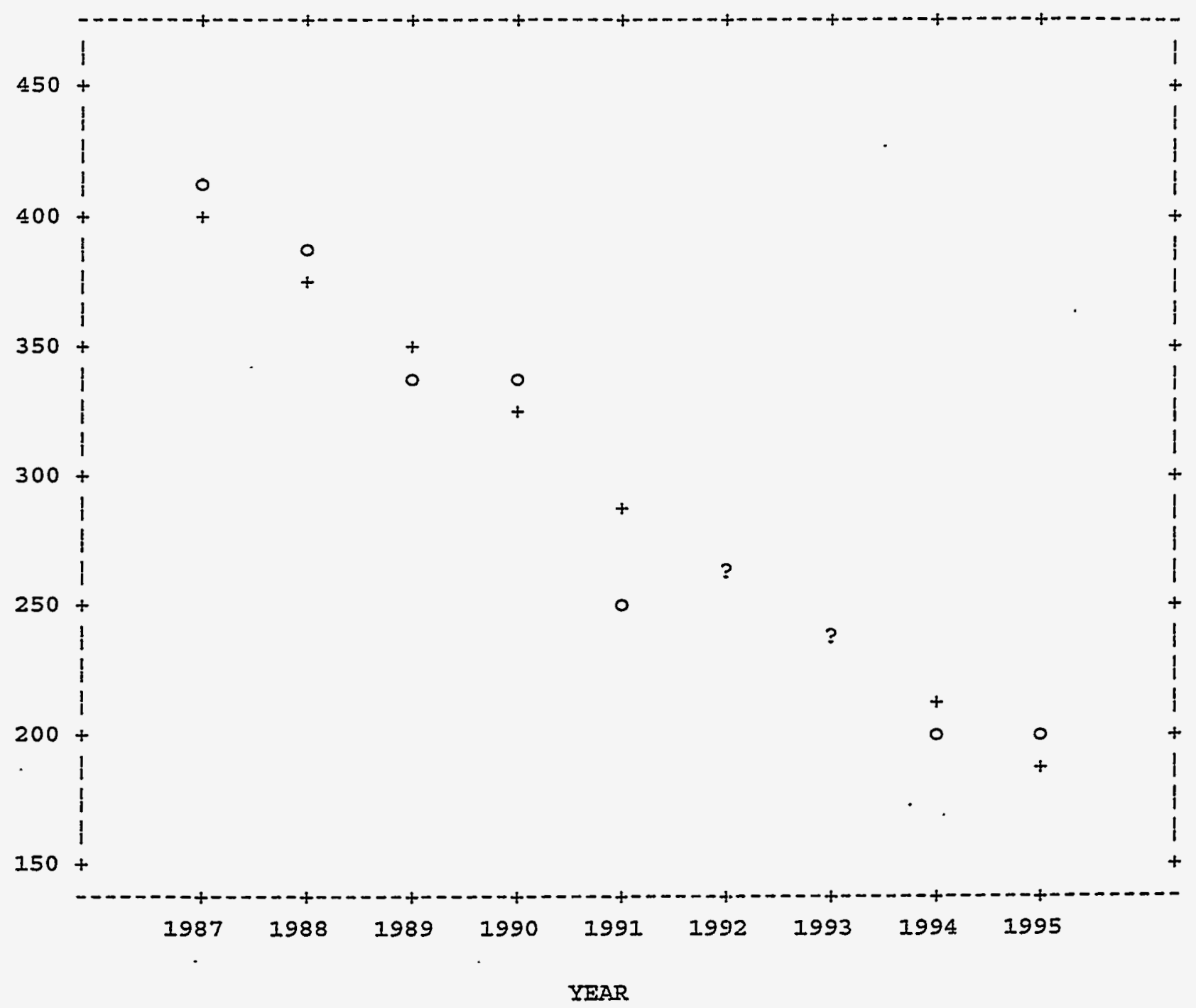


Regression of Radiation Exposure Rate on Calendar Year Rem/plant year vs. Calendar Year

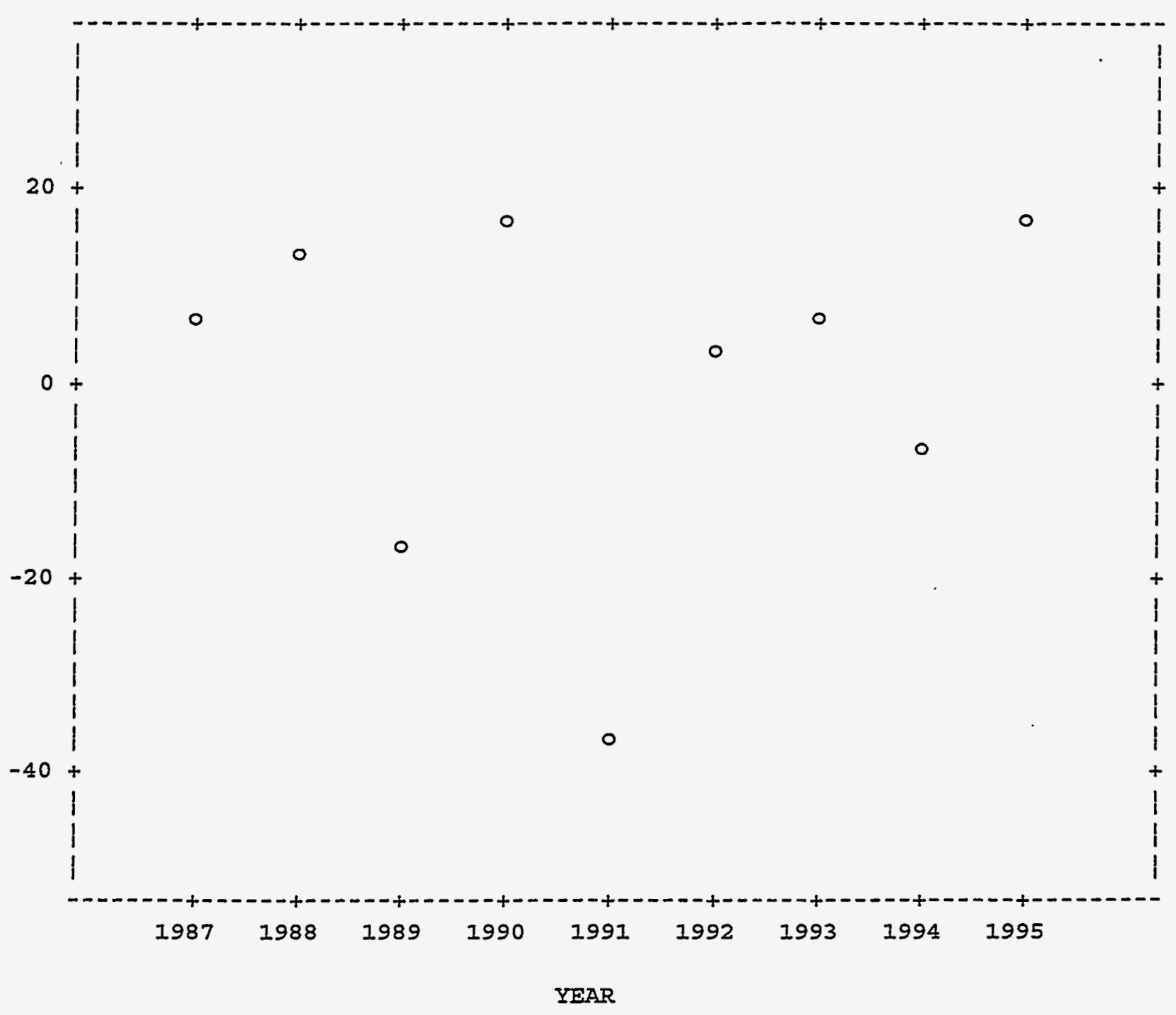


Regression of Radiation Exposure Rate on Calendar Year Rem/plant year vs. Calendar year

Plot of RADNORM*YEAR. Symbol used is ' $O$ '.

plot of RADHAT*YEAR. Symbol used is ' +1 .

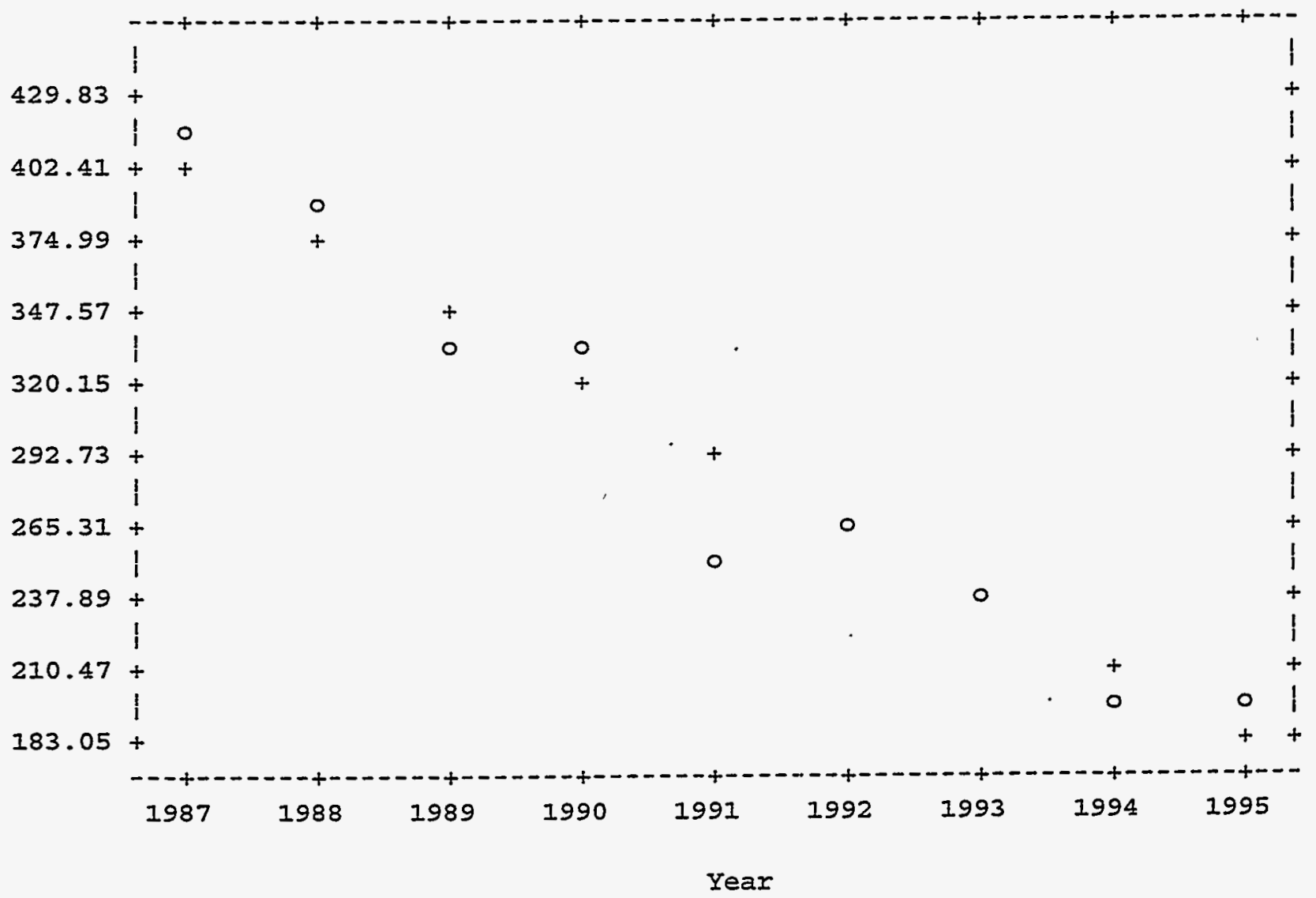

NOTE: 2 obs hidden. 
Plot of RESID*YEAR. Symbol used is ' $O$ '.

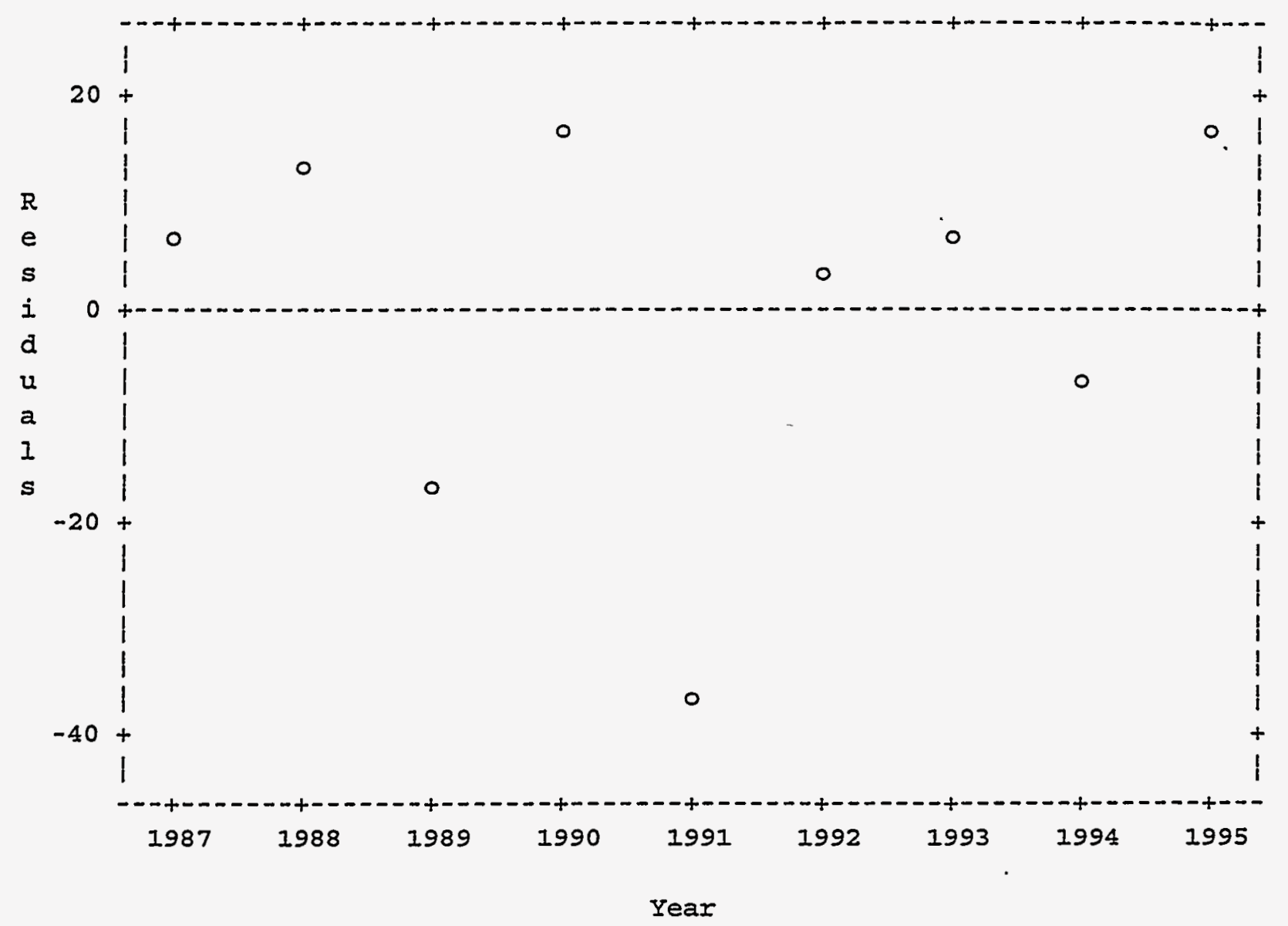


STATA 4.0 Analysis of Example 2 with both one and two covariates;

Model 1:

- regress $\log (\mathrm{N} i / \mathrm{ti}) \quad i$

\begin{tabular}{|c|c|c|c|c|}
\hline source | & ss & MS & Number of obs & $=$ \\
\hline$-\cdots$ & . & 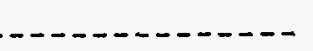 & $F(1, \quad 7)$ & 152.32 \\
\hline Model | & .537963334 & 1.537963334 & Prob > F & $=0.0000$ \\
\hline Residual | & .02472203 & .003531719 & R-squared & 0.9561 \\
\hline$\ldots-\cdots$ & 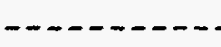 & - - - - - - - - & Adj R-squared & 0.9498 \\
\hline Total & .562685364 & .07033567 & Root MSE & .05943 \\
\hline
\end{tabular}

\begin{tabular}{|c|c|c|c|c|c|c|}
\hline $\log N i / t i \mid$ & Coef. & Std. Err. & $t$ & $P>|t|$ & [95\% Conf. & Interval] \\
\hline--+ & $\cdots-\cdots$ & $\cdots$ & - & & & \\
\hline i 1 & -.0946893 & .0076722 & -12.342 & 0.000 & -.112831 & -.0765475 \\
\hline _cons I & 6.12159 & .0431736 & 141.790 & 0.000 & 6.019501 & 6.22368 \\
\hline
\end{tabular}

Model 2:

- regress $\log (\mathrm{N} i) \quad i$ logti

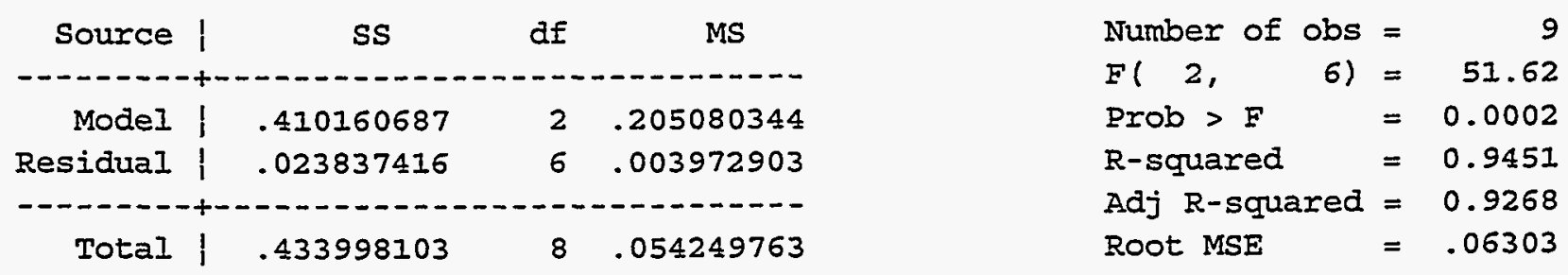

\begin{tabular}{|c|c|c|c|c|c|c|}
\hline $\log N i$ & Coef. & sta. Err. & $t$ & $P>|t|$ & [95\% Conf & IntervaI] \\
\hline & & & 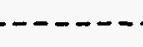 & & 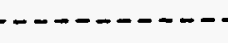 & $-\cdots$ \\
\hline$i \mid$ & -.0905487 & .0119669 & -7.567 & 0.000 & -.1198307 & -.0612668 \\
\hline $\log t i$ & .6650403 & .7098323 & 0.937 & 0.385 & -1.071857 & 2.401937 \\
\hline _cons i & 7.664916 & 3.27087 & 2.343 & 0.058 & -.3386148 & 15.66845 \\
\hline
\end{tabular}

- swilk residl resid2

\begin{tabular}{l|ccccc} 
& \multicolumn{5}{c}{ Shapiro-Wilk $w$ test for normal data } \\
Variable | Obs & $\mathrm{W}$ & $\mathrm{V}$ & $\mathrm{z}$ & $\mathrm{Pr}>\mathrm{z}$ \\
\hline resid1 | & 9 & 0.93026 & 1.025 & 0.041 & 0.48379 \\
resid2 & 9 & 0.96830 & 0.466 & -1.174 & 0.87977
\end{tabular}


Appendix B: Mathematical Details

B-1 
B-2 


\section{Appendix B}

\section{Mathematical Details}

\section{Matrix Notation}

A few basic results will be stated in matrix notation for the purpose of illustration, but the topic will not be fully developed here. In keeping with standard mathematical notation, matrices and vectors will be written in bold type. We will denote the transpose of an arbitrary matrix $A=\left\{a_{i j}\right\}$ by $A^{\mathrm{T}}=$ $\left\{a_{\mathrm{ji}}\right\}$. Furthermore, if $A$ is a square nonsingular matrix then we denote its inverse by $A^{-1}$. Also, we will make no distinction between a $1 \times k$ matrix and a $k$-dimensional row vector. Similarly, a $k \times 1$ matrix will be regarded the same as a $k$-dimensional column vector. Thus, if $c$ represents a $k$-dimensional column vector, then its transpose $c^{\mathrm{T}}$ will represent the corresponding row vector.

\section{Formulation of the Model}

It is possible to write Model (4) and the variance assumptions conveniently in matrix notation. We represent the response variables as an $n \times 1$ column matrix $Y$. We will also use this notation for the column matrix of observed responses $y_{\mathrm{j}}$. The transpose is a $1 \times n$ row matrix $Y^{\mathrm{T}}=\left(Y_{1}, \ldots, Y_{n}\right)$, and we define $\mathrm{E}\left(Y^{\mathrm{T}}\right)=\left(\mathrm{E}\left(Y_{1}\right), \ldots, \mathrm{E}\left(Y_{n}\right)\right)$ and $\mathrm{E}(Y)$ is the corresponding $n \times 1$ column matrix of expected values. Also, we will denote by $V$ a matrix such that the $i j$ th element is the covariance of the variables $Y_{i}$ and $Y_{j}$, $V=\left\{\operatorname{cov}\left(Y_{\mathrm{i}}, Y_{\mathrm{j}}\right)\right\}$. The matrix $V$ is the covariance matrix, and Model (4) can be written as

$E(Y)=X \beta$

and

$V=\sigma^{2} I$

where $X$ is an $n \times(m+1)$ matrix of covariate values $\left\{x_{i j}\right\}$ for $1 \leq i \leq n$ and $0 \leq j \leq m$, and $I$ is the $n \times n$ identity matrix.

\section{Least Squares Estimation}

Equation (5) can be written in matrix notation as

$S(\beta)=(Y-X \beta)^{\top}(Y-X \beta)$. 
The system of Equations (6), which is obtained by taking the partial derivatives of (B-3) with respect to each $\beta_{\mathrm{j}}$ and setting them equal to zero. This system is linear in the $\beta_{\mathrm{j}}$ 's and it is conveniently expressed in terms of the matrix equation.

$X^{\mathrm{T}} Y=X^{\mathrm{T}} X \beta$

Furthermore, if the matrix $X^{\mathrm{T}} X$ is nonsingular, then there exists a unique solution of the form $\hat{\beta}=\left(X^{\mathrm{T}} X\right)^{-1} X^{\mathrm{T}} Y$

We will assume that $X^{\mathrm{T}} X$ is nonsingular. Of course, a more basic assumption would be that $X$ has full rank.

\section{Properties}

The estimators $\hat{\beta}_{j}$ are linear functions of the $Y_{i}$, and it can be shown that they are unbiased. In particular, using (B-1) we have

$$
\begin{aligned}
E(\hat{\beta}) & =\left(X^{\mathrm{T}} X\right)^{-1} X^{\mathrm{T}} \mathrm{E}(Y) \\
& =\left(X^{\mathrm{T}} X\right)^{-1} X^{\mathrm{T}} X \beta \\
& =\beta
\end{aligned}
$$

As in the case of the simple linear model, the LS (and ML) estimators of the $\beta_{j} s$ for the general model are referred to as the best linear unbiased estimators or BLUEs.

It can also be shown that the respective variances and covariances of the BLUEs are the elements of the matrix

$\left\{\operatorname{cov}\left(\hat{\beta}_{\mathfrak{i}}, \hat{\beta}_{\mathfrak{j}}\right)\right\}=\sigma^{2} C$

where $C=\left(X^{\mathrm{T}} X\right)^{-1}=\left\{c_{i j}\right\}$, and that the BLUE of any linear combination of the $\beta_{\mathrm{j}} \mathrm{s}$, say $r \beta$ is given by $r \hat{\beta}$, where $r$ is an arbitrary row vector of known constants. This result is usually called the GaussMarkov Theorem. 


\section{Maximum Likelihood}

The same quantity (B-3) which is minimized to obtain LS estimates is also minimized in solving for the ML estimates of the $\beta_{j} \mathrm{~s}$. It is also possible to write the ML estimator of $\sigma^{2}$, in matrix notation. In particular,

$$
\begin{aligned}
\hat{\sigma}^{2} & =S(\beta) / n \\
& =(Y-\hat{X \hat{\beta}})^{\mathrm{T}}(Y-\hat{X \hat{\beta}}) / n .
\end{aligned}
$$

\section{Confidence Contours}

It can also be shown that

$$
S(\beta)=S(\hat{\beta})+(\hat{\beta}-\beta)^{\mathrm{T}} X^{\mathrm{T}} X(\hat{\beta}-\beta)
$$

The last term on the right of $(B-4)$ is a quadratic form in the differences $\hat{\beta}_{\mathrm{j}}-\beta_{\mathrm{j}}$, which can be written as

$$
Q=\Sigma_{\mathrm{i}} \Sigma_{\mathrm{j}}\left(\hat{\beta}_{\mathrm{i}}-\beta_{\mathrm{i}}\right) d_{\mathrm{ij}}\left(\hat{\beta}_{\mathrm{j}}-\beta_{\mathrm{j}}\right)
$$

where the coefficients $d_{\mathrm{ij}}$ are the elements of the matrix $X^{\mathrm{T}} X$.

\section{Correlated Errors}

The results stated above can be generalized to the case where the covariance assumption given in (B-2) is replace with the assumption

$$
V=\sigma^{2} A
$$

where $A$ is a matrix with known elements $a_{\mathrm{ij}}$. In this situation, the $Y_{\mathrm{i}} \mathrm{s}$ may be correlated and have unequal variances as long as the covariances of $Y_{\mathrm{i}}$ and $Y_{\mathrm{j}}$ are known except for an unknown scale factor $\sigma^{2}$.

It turns out that the BLUEs in this case are obtained by minimizing the weighted sum of squares

$S(\beta)=(Y-X \beta)^{\mathrm{T}} A^{-1}(Y-X \beta)$. 
Note that $\sigma^{2}$ may also be a function of the unknown $\beta_{j} s$, say $\sigma^{2}=h(\beta)$. A result known as the generalized Gauss-Markov Theorem states that the solutions which minimize (B-5) are the BLUEs in this more general setting. The estimates are given by

$\hat{\beta}=\left(X^{\mathrm{T}} A^{-1} X\right)^{-1} X^{\mathrm{T}} A^{-1} Y$

Also, $r \hat{\beta}$ is the BLUE of any linear combination of the $\beta_{j} s$, say $r \beta$ is given by $r \hat{\beta}$, where $r$ is an arbitrary row vector of known constants. It is interesting to note that if $I$ is used in place of $A$, the ordinary LS estimates are unbiased, but they are not the BLUE's in this case because they are not weighted properly. 